\title{
Measurement of the electron transmission rate of the gating foil for the TPC of the ILC experiment
}

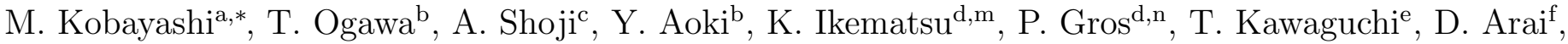 \\ M. Iwamura ${ }^{f}$, K. Katsukif ${ }^{\mathrm{f}}$, A. Kotof ${ }^{\mathrm{f}}$, M. Yoshikaif ${ }^{\mathrm{f}}$ K. Fujii ${ }^{\mathrm{a}}$, T. Fusayasu ${ }^{\mathrm{d}}$, Y. Kato ${ }^{\mathrm{g}}$, S. Kawada ${ }^{\mathrm{h}}$, \\ T. Matsuda ${ }^{\mathrm{a}}$, S. Narita ${ }^{\mathrm{c}}$, K. Negishic ${ }^{\mathrm{c}}$,H. Qi ${ }^{\mathrm{i}}$, R.D. Settles ${ }^{\mathrm{j}}$, A. Sugiyama ${ }^{\mathrm{d}}$, T. Takahashi ${ }^{\mathrm{e}}$ J. Tian $^{\mathrm{a}, \mathrm{o}}$, \\ T. Watanabe ${ }^{\mathrm{k}}, \mathrm{R}$. Yonamine ${ }^{\mathrm{l}}$ \\ ${ }^{a}$ High Energy Accelerator Research Organization (KEK), Tsukuba 305-0801, Japan \\ ${ }^{b}$ The Graduate University for Advanced Studies (Sokendai), Tsukuba 305-0801, Japan \\ ${ }^{c}$ Iwate University, Iwate 020-8551, Japan \\ ${ }^{d}$ Saga University, Saga 840-8502, Japan \\ ${ }^{e}$ Hiroshima University, Higashi-Hiroshima 739-8530, Japan \\ ${ }^{f}$ Fujikura Ltd., 1440, Mutsuzaki, Sakura-city, Chiba 285-8550, Japan \\ ${ }^{g}$ Kindai University, Higashi-Osaka 577-8502, Japan \\ ${ }^{h}$ Deutsches Elektronen-Synchrotron (DESY), D-22607 Hamburg, Germany \\ ${ }^{i}$ Institute of High Energy Physics, Chinese Academy of Sciences, Beijing 100049, China \\ ${ }^{j}$ Max Planck Institute for Physics, DE-80805 Munich, Germany \\ ${ }^{k}$ Kogakuin University, Shinjuku 163-8677, Japan \\ ${ }^{l}$ Department of Physics, Tohoku University, Sendai 980-8578, Japan \\ ${ }^{m}$ now at Institute of Multidisciplinary Research for Advanced Materials (IMRAM), Tohoku University, Sendai 980-857r, \\ Japan \\ ${ }^{n}$ now at Department of Physics, Engineering Physics 83 Astronomy, Queen's University, Kingston, Ontario K' 7 L $3 N 6$, \\ Canada \\ ${ }^{\circ}$ now at ICEPP, University of Tokyo, Hongo, Tokyo 113-0033, Japan
}

\begin{abstract}
We have developed a gating foil for the time projection chamber envisaged as a central tracker for the international linear collider experiment. It has a structure similar to the Gas Electron Multiplier (GEM) with a higher optical aperture ratio and functions as an ion gate without gas amplification. The transmission rate for electrons was measured in a counting mode for a wide range of the voltages applied across the foil using an ${ }^{55} \mathrm{Fe}$ source and a laser in the absence of a magnetic field. The blocking power of the foil against positive ions was estimated from the electron transmissions.
\end{abstract}

Keywords:

Ion gating, Electron transmission, Positive ions, TPC, ILC

PACS: 29.40.Cs, 29.40.Gx

\section{Introduction}

We are designing a time projection chamber (TPC) [1-3] using gas electron multipliers (GEMs) [4] or Micromegas [5] for the central tracker of the international large detector (ILD) [6] for the future international linear collider (ILC) experiment [7]. A high momentum resolution of $\sigma_{P_{\mathrm{t}}} / P_{\mathrm{t}} \sim 10^{-4} \cdot P_{\mathrm{t}}[\mathrm{GeV} / \mathrm{c}]$ is required of the ILD-TPC alone under an axial magnetic field of $\sim 3.5 \mathrm{~T}$. In order to fulfill the requirement

*Corresponding author. Tel.: +8129864 5376; fax: +81298642580.

Email address: makoto.kobayashi.exp@kek.jp (M. Kobayashi) 
within the space available for the TPC, each readout pad row with a height of $5-6 \mathrm{~mm}$ needs to have azimuthal spatial resolution of $\lesssim 100 \mu \mathrm{m}$ for stiff radial tracks throughout the entire sensitive volume.

It is difficult to achieve such high spatial resolution in a strong magnetic field with conventional readout modules (sectors) equipped with a multi-wire proportional chamber (MWPC) due to the so-called $E \times B$ effect near the wire planes [8]. Among micro-pattern gas detectors (MPGDs), which are virtually free from the $E \times B$ effect as well as from the angular wire effect, our group (ILD-TPC Asia) has chosen GEM as a gas amplification device to be operated in an appropriate gas mixture because of the natural charge spread in its stack during gas multiplication.

The azimuthal spatial resolution of a GEM-based TPC for a single pad row is given by

$$
\sigma_{\mathrm{X}}^{2}=\sigma_{\mathrm{X} 0}^{2}+\frac{D_{\mathrm{T}}^{2} \cdot z}{N_{\mathrm{eff}}}
$$

where $\sigma_{\mathrm{X} 0}$ is a constant term, $D_{\mathrm{T}}$ denotes the transverse diffusion constant of drift electrons, $z$ is the drift distance, and $N_{\text {eff }}$, the effective number of electrons [9 14]. For tracks normal to the pad row Eq. (1) reduces to

$$
\sigma_{\mathrm{X}}^{2}=\frac{1}{N_{\mathrm{eff}}} \cdot\left(B_{0}^{2}+D_{\mathrm{T}}^{2} \cdot z\right)
$$

where $B_{0}$ is a constant determined by the pad pitch ( $\sim$ pad width) and the width of charge spread in the gas amplification device (a GEM stack in our case) [11] The spatial resolution of around $100 \mu \mathrm{m}$ seems feasible with a gas mixture of $\operatorname{Ar}-\mathrm{CF}_{4}(3 \%)$-isobutane $(2 \%)$ [11, 15, 16].

The major remaining issue to be addressed is the influence of positive ions accumulated in the drift volume of the TPC. The positive ions disturb the otherwise uniform drift field and distort the reconstructed tracks. The ILC machine has a peculiar time structure of the beam crossings. The electron and positron beams are both a train of 1312 bunches, 554 nsec apart in time (baseline design), and collide every $200 \mathrm{msec}$ with a duration of $\lesssim 1 \mathrm{msec}$ [17]. According to the estimation based on a simulated beam background [18], the influence of primary ions is expected to be small, whereas that of secondary ions created in the gas amplification is not acceptable [6]. The secondary ions form a $\sim 3$-mm-thick disk above the gas amplification device just after each beam (bunch-train) crossing. The ion disk then slowly drifts back towards the central membrane with small diffusion. As a result, $3-4$ ion disks $(\sim 75 \mathrm{~cm}$ apart, depending on the drift field and the mobility of positive ions) co-exist in the drift volume at the end of every beam crossing ${ }^{2}$.

Each ion disk and the corresponding negative charges induced on the surface of the field cage create a radial electric field $\left(E_{\mathrm{r}}\right)$ around the disk, dependent on its location along the detector axis $(z)$. The radial electric field in turn causes azimuthal deflection of the primary electrons during their drift towards the readout plane by the $E \times B$ effect. The total azimuthal displacement (offset) of a drift electron due to the ion disks depends on its created position $(r, z \text {, and possibly } \phi)^{3}$. See Ref. [6] and references cited therein for details.

It should be noted that Fig. III-2.15 in Section 2.3.1.7 (right panel) of Ref. [6] shows the azimuthal displacement due to a single ion disk with its charge density estimated assuming a small amount of ion

\footnotetext{
${ }^{1}$ The coordinate offset (bias) at short drift distances intrinsic to the barycenter method for the hit point reconstruction in a pad row is assumed to have been properly removed.

${ }^{2}$ The positive ions are assumed to be iso- $\mathrm{C}_{4} \mathrm{H}_{10}{ }^{+}$. The figures in Ref. [19] suggest that the dominant (final) positive ions are most likely hydrocarbons, at least in the case of binary mixtures of $\mathrm{CF}_{4}$ and a hydrocarbon.

${ }^{3}$ The displacement is largest at the maximum drift distance as expected, and at the minimum detector radius because the center of gravity of the beam-induced background, and therefore that of charge density within the ion disk is closer to the inner field cage.
} 
backflow from the amplification device: one outgoing ion per incoming drift electron. The fractional ion backflow of the gas amplification device (IBF) is often defined as the average ratio of the number of outgoing positive ions to the number of amplified electrons reaching the readout pad plane for a single incoming drift electron ${ }^{4}$. The planned effective gas gain is 2000 and the value of IBF is expected to be $\lesssim$ $5 \%$ for our present double-GEM configuration ${ }^{5}$. Consequently, the actual azimuthal displacement could be about $2 \times 100$ times that shown in the figure for a single ion disk, resulting in the maximum total displacement of $\mathcal{O}(1 \mathrm{~mm})^{6}$. In addition, the estimation of the background hits in the TPC takes into account only the electron-positron pairs created by the beam-induced two-photon process, assuming a set of beam parameters for $\sqrt{s}=500 \mathrm{GeV}[18]$.

We plan to operate the TPC in a semi-continuous mode with a gating plane, exploiting the beam timing structure mentioned above, in order to prevent the secondary ions from entering the drift volume while keeping it fully active during the beam collisions. The gating plane is placed $\lesssim 10 \mathrm{~mm}$ above the gas amplification device. The gate remains open during the beam crossing and is closed after collecting all the drift electrons. The back-drifting ions are almost completely trapped by the gating plane within several milliseconds after its closure. The gate is then re-opened prior to the next beam crossing. As a consequence, there are no ion disks at the beginning of each beam crossing. The influence $\left(E_{\mathrm{r}}\right)$ of the ions building up below the gating plane during the beam crossing is expected to be small since those ions couple to the induced charges nearby on the surface of the amplification device and the gating plane (with a fine mesh structure), by the electric field lines almost parallel with the detector axis, except for the small region near the field cage.

Inspired by the work by F. Sauli and coworkers [20] we have developed a gating plane without wires after a detailed simulation study [21] and experiments with small prototypes [22]. It has a thin GEMlike structure with a large optical aperture ratio, but is operated without gas amplification. The large aperture ratio is required to ensure good electron collection efficiency under a high axial magnetic field while the thin insulator (polyimide) is favorable to reduce the possibility for drift electrons in the hole to be (temporarily) trapped on its surface. The hole pitch is determined by the required aperture ratio ( $\gtrsim 80 \%$ ) and the minimum possible rim width of the applied fabrication technique with a reasonable yield. It should also be noted that the smaller the hole pitch the lower is the switching voltage of the gating plane needed to make it opaque to positive ions.

It is essentially a precisely aligned double-mesh layer with an embedded insulating spacer in-between, to be referred to as the gating foil hereafter in the present paper. The gating foil with a frame can be mounted on top of the amplification GEM stack or Micromegas, with either ordinary pad readout or pixel readout [23, 24], just like an additional ordinary GEM foil, thereby realizing a wire-less gas amplification device for TPCs with ion-blocking capability 7 .

In addition to the high blocking capability against positive ions at the closed state, the gating foil is required to have high transparency of $\gtrsim 80 \%$ for drift electrons at the open state in order not to compromise appreciably the azimuthal spatial resolution particularly at long drift distances since $N_{\text {eff }}$ in Eqs. (1) and (2) is approximately proportional to the average number of drift electrons per pad row.

\footnotetext{
${ }^{4}$ The contribution of primary ions is excluded.

${ }^{5}$ The GEM stack is not optimized in terms of low IBF. Geometrical parameters of the GEM foils and/or voltage settings of the GEM stack affecting the electron transmission, therefore compromising $N_{\text {eff }}$, are ruled out in our case.

${ }^{6}$ The displacement of drift electrons is caused mainly by the two ion disks in the middle part of the drift volume since the disk located near the readout plane or the central membrane contributes little (see Fig. III-2.15 in Ref. [6]).

${ }^{7}$ A sense-wire-less TPC with anode-pad readout was proposed by H.J. Hilke, well before the invention of Micromegas, with its advantages over conventional MWPC readout [25. A similar TPC employing a PPAC (Parallel Plate Avalanche Chamber) as a gas amplification device was investigated by A. Peisert [26].
} 
In this paper we present the electron transmission rate of a real-size gating foil measured using an ${ }^{55} \mathrm{Fe}$ source and a laser for a wide range of the voltages applied across the foil in the absence of a magnetic field. A brief description of the gating foil is given in the next section. The experimental setup and the data taking procedure are described in Section 3, and the results are presented in Section 4. Section 5 is devoted to discussions on the results, and Section 6 concludes the paper along with future prospects.

\section{Gating foil}

The gating foil was fabricated by Printed Circuit Technology R\&D Department of Fujikura Ltd. using dedicated flexible printed-circuit production techniques in order to realize a thin GEM-like structure having hexagonal holes with narrow rims [27].

First, a fine honeycomb-shape pattern is formed on the front side of a copper-clad polyimide film (12.5 $\mu \mathrm{m}$ thick) by photolithography with a single mask. Next, hexagonal holes are bored in polyimide by a de-focused UV-YAG laser beam with the patterned copper layer as a mask. Finally, the copper layer on the back side at the bottoms of the hexagonal holes is etched away from the both sides, without (ferromagnetic) nickel-plating for the protection of the copper pattern on the front side. A honeycomb-shape pattern of the copper clad on the back side, which is well aligned with that on the front side, is formed in the final process by exploiting the difference in the etching speed. It should be emphasized that the precise alignment of the fine patterns on the both sides is technically difficult with double-mask photolithography. See Ref. [27] for the technical details of the production.

Fig. 1 shows the completed gating foil. The size of the nearly trapezoidal effective area is $\sim 143 \mathrm{~mm}$ $\times(211 \mathrm{~mm}-232 \mathrm{~mm})$, which fits in our prototype of the ILD-TPC readout module. The hole pitch is 335 $\mu \mathrm{m}$ and the rim width is $\sim 26 \mu \mathrm{m}(\sim 31 \mu \mathrm{m})$ on the front (back) side, yielding an optical aperture ratio of $\sim 82 \%$. Magnified photos of the foil are shown in Fig. 2 while Fig. 3 shows the cross section of the rim. The typical error of the dimensions shown in the figures is $\lesssim \pm 1 \mu \mathrm{m}$.

\section{Experimental setup and data taking}

\subsection{Setup}

The experiment was conducted after the measurement of the avalanche fluctuation [28] with essentially the same setup using a test chamber box for the prototype readout module of the ILD-TPC, and a radiation source: an un-collimated ${ }^{55} \mathrm{Fe}$ source or a laser. Only the amplifier and the digitizer (modified ALTRO readout system [29, 30]) were replaced with the combination of a charge-sensitive preamplifier (ORTEC 142PC), a shaper amplifier (ORTEC 672), and a CAMAC peak-sensitive analog-to-digital converter module (Hoshin ADC C008).

The experimental setup is schematically shown in Fig. 4. The test chamber box contains a double GEM stack, a readout plane right behind it, a cathode mesh, and the gating foil to be tested on top of the GEM stack with the front side facing the drift region 8 . The GEM foil is a copper-clad liquid crystal polymer (LCP) with holes drilled by a laser [31, supplied by Scienergy Co. Ltd. The thickness of the LCP is $100 \mu \mathrm{m}$ and the hole diameter (pitch) is $70 \mu \mathrm{m}(140 \mu \mathrm{m})$. The readout plane is paved over with 28 pad rows (5.26 mm wide), each consisting of small pads arranged at a pitch of $\sim 1.2 \mathrm{~mm}$. The adjacent pad rows are staggered by half a pad pitch. The transfer and induction gaps of the double GEM stack are $4 \mathrm{~mm}$ and $2 \mathrm{~mm}$, respectively. The distance between the gating foil and the GEM stack is $9.4 \mathrm{~mm}$, and is referred to hereafter as the transfer region (see Fig. 5).

\footnotetext{
${ }^{8}$ Actually, the GEM stack is set upside down in the setup, and the gating foil is placed below it (see Fig. 4).
} 


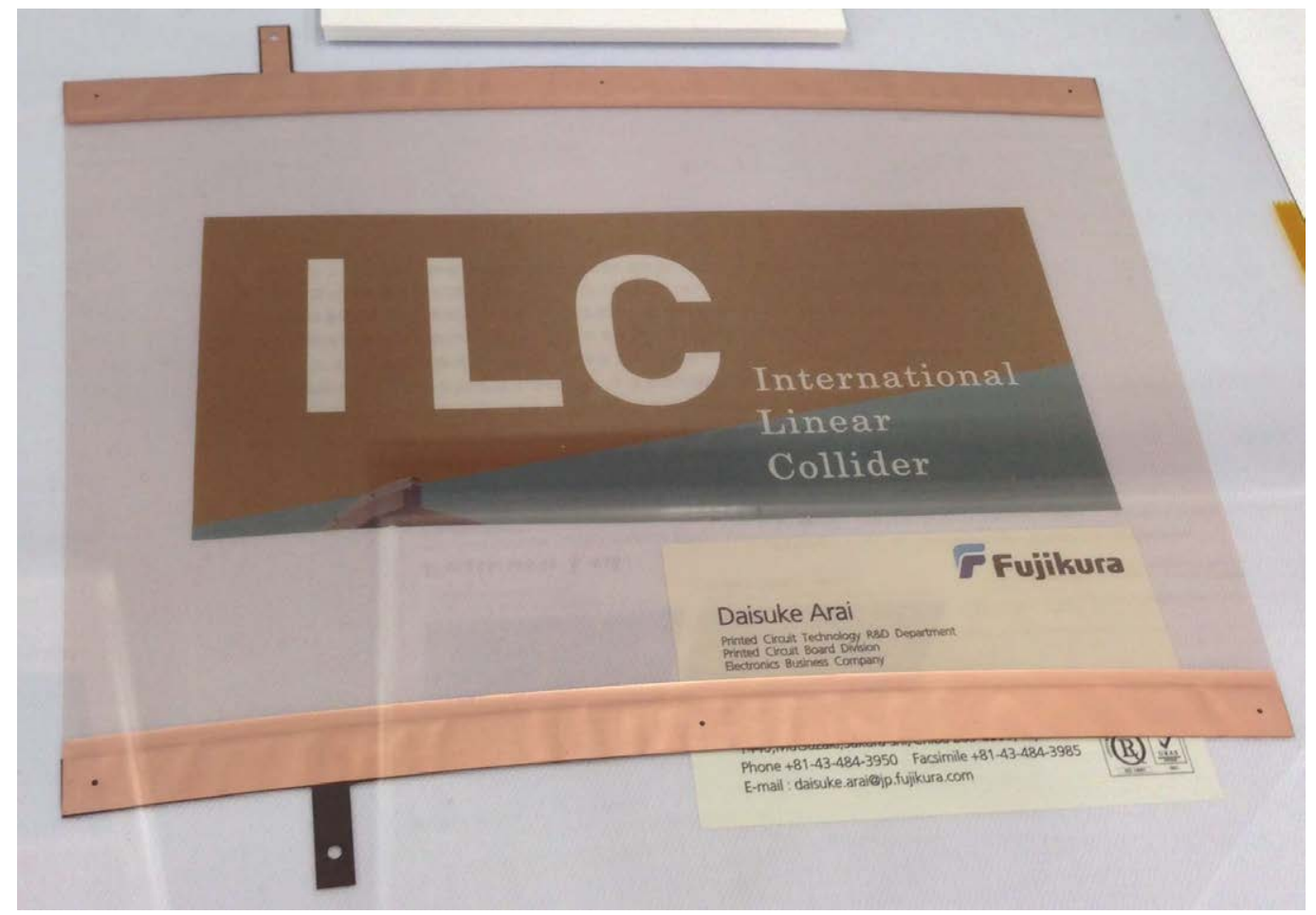

Figure 1: Photograph of the gating foil. The effective area is almost invisible because of its high optical transparency.
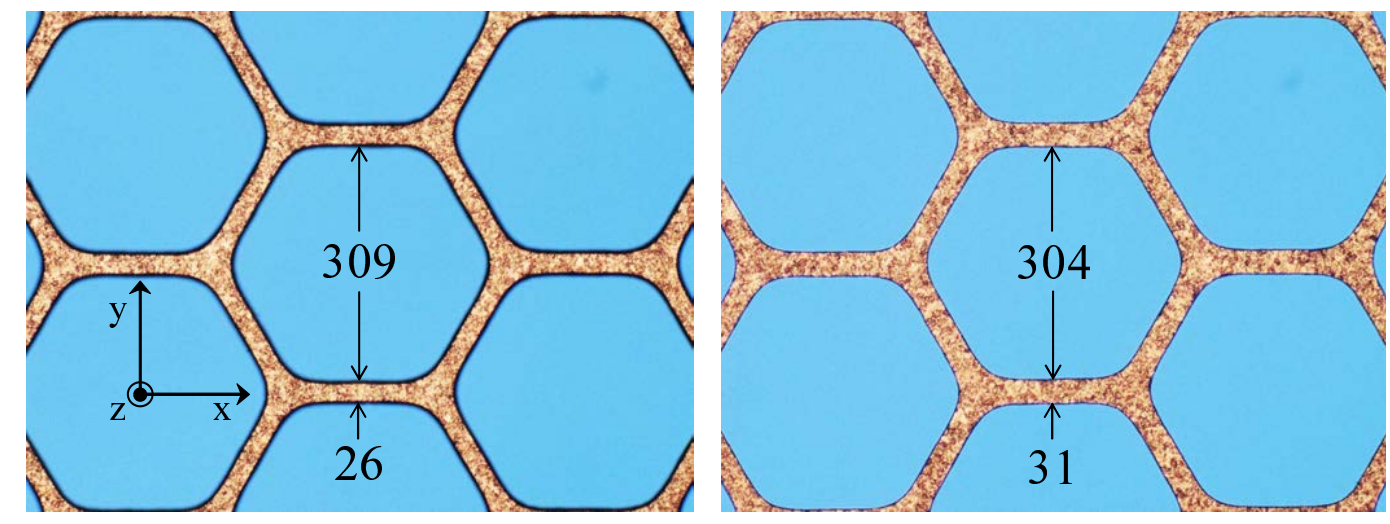

Figure 2: Magnified photographs of the effective area of the gating foil: the left (right) panel for the front (back) side, with dimensions in microns. 


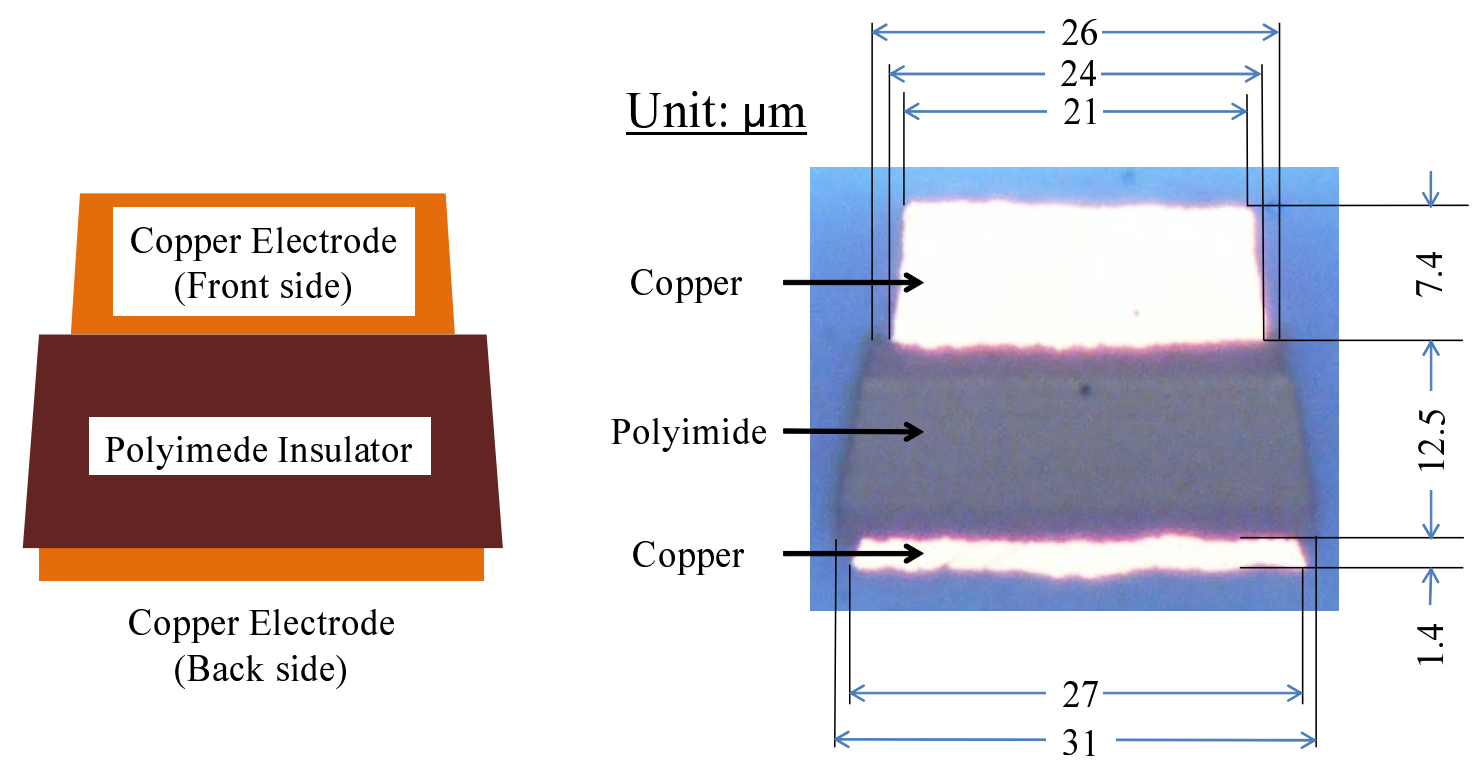

Figure 3: Cross-section of the hexagonal rim in the effective area of the gating foil: a schematic view (left) and a photomicrograph with the typical dimensions (right). The difference in thickness between the upper and lower electrodes is due to the fabrication technique applied.

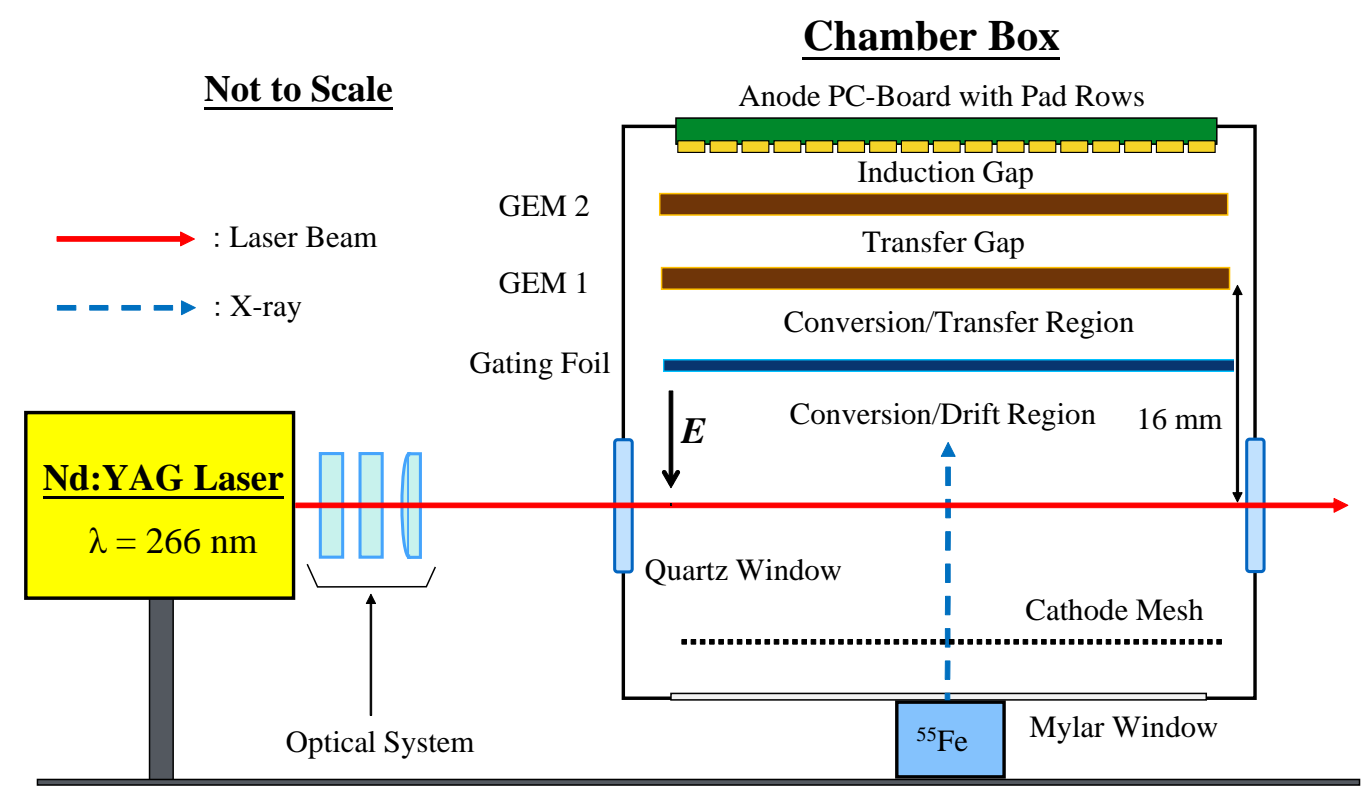

Figure 4: Schematic view of the experimental setup. 


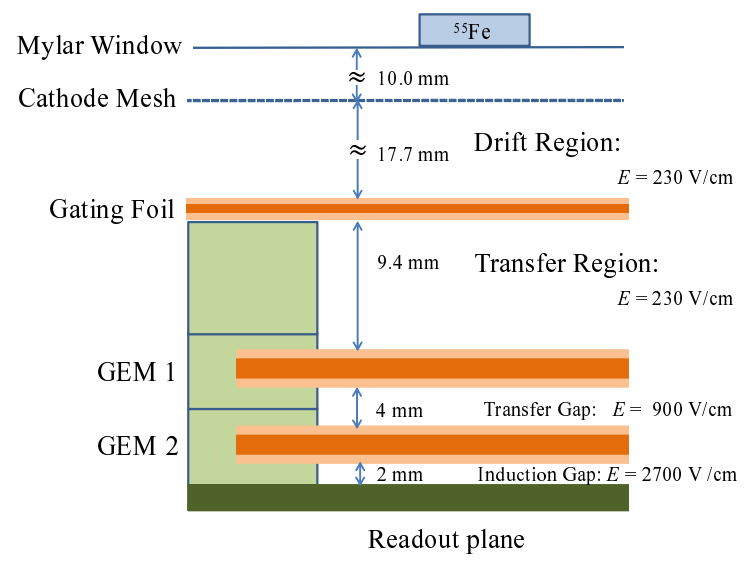

Figure 5: Schematic view of the GEM stack and the gating foil. The actual setup is flipped, with the ${ }^{55}$ Fe source placed at the bottom (see Fig. 4).

In the previous experiment, individual signals induced on the pads were read out through multi-contact connectors arranged on the back side of the readout plane. In the present setup, on the other hand, the signal charges on the pads located at the area under study are summed on the corresponding connector (central connector), and then sent to a preamplifier located nearby via a coaxial cable. Thus, the signal charge is measured on a connector-by-connector basis, instead of a pad-by-pad basis in the previous experiment. Each connector reads out signals on 32 pads in adjacent two pad rows, covering an area of $\sim$ $11 \mathrm{~mm} \times 19 \mathrm{~mm}$ on the pad plane. Fig. 6 shows the pads' side and the connectors' side of the readout plane. The charges on the connectors directly surrounding the central connector are summed on the spot. The analog sum is then sent to another preamplifier in the measurement with the ${ }^{55} \mathrm{Fe}$ source in order to select off-line the events in which most of the charge is collected on the central connector (see Section 4.1). All the remaining pads are grounded on the connectors.
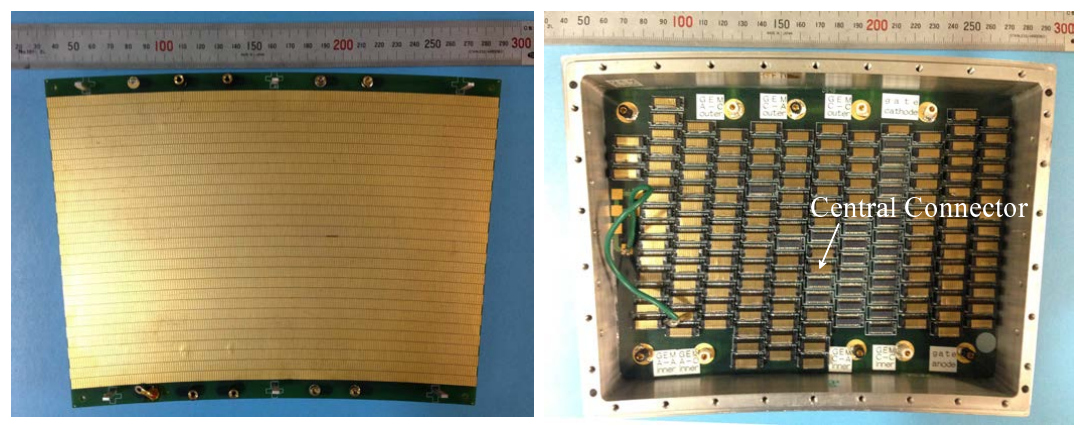

Figure 6: Photograph of the pads' side (left) and the connectors' side (right) of the readout plane. The location of the central connector is indicated by an arrow. The analog sum of the signals on the connectors directly surrounding it is also recorded (see text).

In the measurement with the laser (New Wave Research Polaris II), a pulsed UV beam $(\lambda=266 \mathrm{~nm})$ is injected into the chamber box, located at the laser focal point, through a quartz window. The pulse duration is $3-4 \mathrm{nsec}$ and the repetition rate is $20 \mathrm{~Hz}$. The laser beam size $(2 \sigma)$ is about $600 \mu \mathrm{m}(300$ $\mu \mathrm{m})$ at the focal point, in the horizontal (vertical) direction. Electrons are created along the beam by two-photon ionization processes and detected by the pad rows arranged normally to the beam after gas 
amplification. Only the signals on the central connector are sent to the preamplifier and used in the analysis.

The event trigger and the ADC gate are generated by the output signal of the preamplifier for the central connector with a sufficiently low discriminator threshold (self-trigger) in the ${ }^{55} \mathrm{Fe}$ runs, while in the laser runs the external trigger is provided by the laser as a logic signal synchronized to its firing. The width of the gate provided to the ADC is 500 nsec with its center located at the signal-peak timing both in the ${ }^{55} \mathrm{Fe}$ runs and laser runs. The relatively narrow gate as compared to the amplified signal width of $\gtrsim 12$ usec (FWHM) assures that the peak-sensitive ADC output always contains the signal contribution (if any) along with the electronic noise component just below the signal peak. This is crucial in the laser runs with the gating foil almost opaque to the incoming electrons? The schematic diagram of the readout electronics is shown in Fig. 7. The total gain of the amplifiers is $14 \mathrm{~V} / \mathrm{pC}$ and $10 \mathrm{~V} / \mathrm{pC}$, respectively in the ${ }^{55} \mathrm{Fe}$ runs and the laser runs. The CAMAC ADC has 12-bit resolution for $0-2.5 \mathrm{~V}$ input. The signal charges recorded by the ADC are sent to a Linux PC via Ethernet.
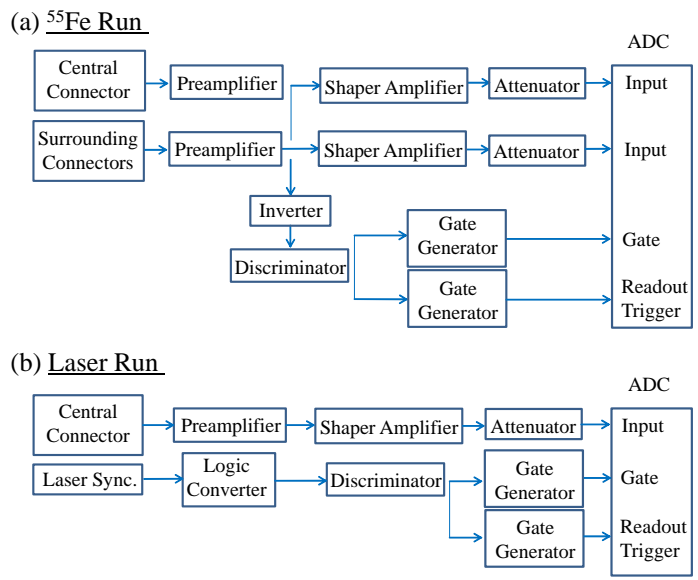

Figure 7: Schematic diagrams of the readout electronics for the ${ }^{55} \mathrm{Fe}$ runs (a) and the laser runs (b).

The filling gas is a premix of $\mathrm{Ar}-\mathrm{CF}_{4}(3 \%)$-isobutane $(2 \%)$ at atmospheric pressure and room temperature $\left(\sim 25^{\circ} \mathrm{C}\right)$. The typical flow rate of the gas is $200 \mathrm{cc}$ per minute, corresponding to about 4 flushes of the chamber box volume per hour. The high voltage applied across the first (second) stage GEM is $345 \mathrm{~V}$ (315 $\mathrm{V}$ ) and the electric field in the transfer (induction) gap is $900 \mathrm{~V} / \mathrm{cm}(2700 \mathrm{~V} / \mathrm{cm}$ ), yielding a gas gain of $\sim 3700$. The electric fields in the drift region $\left(E_{\mathrm{d}}\right)$ and the transfer region $\left(E_{\mathrm{t}}\right)$ are both set to $230 \mathrm{~V} / \mathrm{cm}$.

\subsection{Data taking}

First, the electron transmission rate was measured with the ${ }^{55} \mathrm{Fe}$ source using the technique employed in Ref. [20]. A set of runs, one with the normal drift field (normal run) and the other with the reversed field (reverse run), was repeated for each voltage across the gating foil $(\Delta V)$. The electric field in the transfer region $\left(E_{\mathrm{t}}\right)$ was always kept at $230 \mathrm{~V} / \mathrm{cm}$. The normal run gives the pulse height distribution for the photons converted in the drift region (gated signals) as well as for those converted in the transfer region (ungated signals) while the reverse run provides the distribution only for the ungated signals. The data taking time of each run was 10 minutes. In the analysis, the distribution of the reverse run was subtracted from that of the normal run in order to obtain the precise photo-peak position for the gated electrons.

\footnotetext{
${ }^{9}$ The main contributor to the noise is the small and slow tail part of the laser-induced electronic noise synchronized to each laser shot, accompanied by a relatively fast ringing component $(\lesssim 50 \mathrm{kHz})$. The noise level varies slightly over time and is monitored by frequent pedestal runs with the optical shutter of the laser closed (see Sections 3.2 and 4.2).
} 
This procedure was required especially when the transmission rate of the gating foil was high and the four peaks (including escape peaks) partially overlapped in the normal run. The data were taken for $\Delta V$ between $-4.5 \mathrm{~V}$ and $+19.5 \mathrm{~V}{ }^{10}$. The negative sign of $\Delta V$ indicates the electric field direction within the holes of the gating foil opposite to the drift field. For $\Delta V$ less than $-4.5 \mathrm{~V}$ reliable measurements of the photo-peak position were difficult because of electronic noise.

The transmission rate was measured for $\Delta V$ down to $-15.5 \mathrm{~V}$ using the laser with a larger number of signal electrons. The laser beam could be injected only into the drift region, i.e. upstream of the gating foil in the present setup. Therefore, runs for the normalization of the transmission rates were necessary at $\Delta V$ where the transmission rate had been measured in the ${ }^{55} \mathrm{Fe}$ runs.

In the data-taking with the laser a set of three runs was repeated for each $\Delta V$ : a run with a value of $\Delta V$ under study, a run with $\Delta V=-0.5 \mathrm{~V}$ for the normalization, and a pedestal run with the optical shutter of the laser closed. The frequent pedestal runs were necessary in particular at highly negative $\Delta V$ s to monitor closely the position and the width of the pedestal, which were found to vary slightly over time because of laser-induced electronic noise synchronized to the shots, and therefore to the signals (see footnote 9). In the analysis the average was taken for the peak positions measured in the two normalization runs, just before and after the run for $\Delta V$ under study since the ionization density by the laser beam was found to decrease slowly and monotonically over time, in addition to tolerable shot-to-shot fluctuations.

The typical data taking time of each run was 10 minutes, corresponding to 12000 laser shots. It was short enough to ensure the constant gas density, and therefore the gas gain of the GEM stack, during each set of three runs.

The pedestal of the ADC (its position and width) was periodically measured during the data taking with the ${ }^{55} \mathrm{Fe}$ source as well, whereas the linearity of the amplifier response to test pulses was checked at the beginning and the end of a series of runs. Appreciable long term charging-up effect was not observed for the gating foil as well as for the amplification GEM stack throughout the experiment.

The measurements of the transmission rate were conducted with the central connector located in the middle of the readout plane after its uniformity had been confirmed by a scan with the ${ }^{55}$ Fe source over the effective area of the gating foil, at a fixed $\Delta V$.

\section{Results}

\subsection{Measurement with ${ }^{55} \mathrm{Fe}$ source}

We used the events for which most of the charge was collected by the central connector. The remaining charges on the surrounding connectors were added after corrected for the slight difference in the amplifier gain. Several examples of the pulse height spectra obtained in the ${ }^{55} \mathrm{Fe}$ runs are shown in Fig. 8 for the events in which more than $95 \%$ of the total charge is deposited on the central connector 11 . It is clear that some portion of drift electrons is captured by the gating foil, depending on $\Delta V$, for the photons converted in the drift region (gated signals). The electron transmission rates were obtained from the ratio of the photo-peak positions (the gated to the ungated) determined by Gaussian fittings after pedestal subtraction for $-4.5 \mathrm{~V} \leq \Delta V \leq+19.5 \mathrm{~V}$. The photo-peak was fitted with a single Gaussian although it contained two

\footnotetext{
${ }^{10} \mathrm{~A}$ half volt $(0.5 \mathrm{~V})$ often appears for the value of $\Delta V$ since the outputs of the high-voltage power supply for the gating foil were found to be slightly different from the set (nominal) values after the measurements. The resultant offset to $\Delta V$ was measured to be $-0.5 \mathrm{~V}$ with a high precision electrometer. The transmission rate for $\Delta V=0 \mathrm{~V}$ was measured with the electrodes of the gating foil short-circuited.

${ }^{11}$ The signals from the surrounding connectors contained electronic noise larger than that from the central connector, mainly because the raw signals from many connectors were put together on the backside of the readout plane, to be fed to the single preamplifier. The resolution of the photo-peak is therefore better with a larger fraction of the signal on the central connector, but at the sacrifice of statistics.
} 


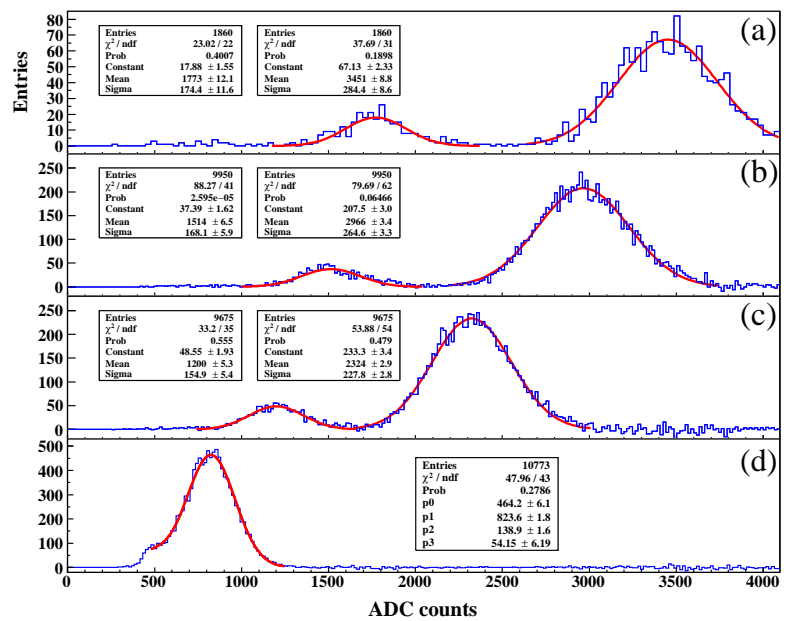

Figure 8: Examples of the pulse height distributions in the ${ }^{55} \mathrm{Fe}$ runs: (a) for the ungated signals, and (b), (c), (d) for the gated signals obtained respectively with $\Delta V=+3.5 \mathrm{~V}, 0.0 \mathrm{~V}$ and $-4.5 \mathrm{~V}$, along with the fitted Gaussians. Most of the escape-peak signals are discriminated by the hardware trigger for $\Delta V=-4.5 \mathrm{~V}$, and a double Gaussian was fitted with its peak position and width relative to those of the photo-peak being fixed. The pedestal is located around 110 ADC counts.

Gaussians corresponding to $\mathrm{Mn}_{\alpha}$ and $\mathrm{K}_{\beta}$ photons since the latter were not suppressed with a chromium filter in the present experiment. The ratio of the numbers of converted photons $\left(\mathrm{K}_{\beta}\right.$ to $\left.\mathrm{K}_{\alpha}\right)$ depends on the distance from the source. In the present setup, however, the average energies are almost identical for photons converted in the drift region and those converted in the transfer region.

The results are shown in Fig. 9 along with those obtained with the laser, and those given by simulation (see Section 4.2). The obtained transmission rates were found to be almost insensitive to the fraction of the signal required on the central connector.

Only the photo-peak positions were used for the measurement of the electron transmission rate. It is interesting, however, that the energy resolution (width) as a function of the transmission rate can be used, in principle, to determine the Fano factor and the size of avalanche gain fluctuation for single drift electrons independently if the exact average number of primary electrons (or the $W$-value) and the collection efficiency of the first GEM are known. The measurement principle is briefly described in Appendix along with a preliminary result.

\subsection{Measurement with laser}

In the laser runs the charge only on the central connector was recorded. The typical number of electrons created along a laser-induced track is 1300 for a single connector (two pad rows). Examples of the pulse height distributions are shown in Fig. 10 along with that obtained with a normalization run at $\Delta V=$ $-0.5 \mathrm{~V}$. The electron transmission rate for each $\Delta V$ was determined from the ratio of the peak position to that in the corresponding normalization run, for which the transmission rate had been measured to be $58.74 \pm 0.11 \%$ using the ${ }^{55} \mathrm{Fe}$ source.

The peak position was defined as the simple average of the pulse height distribution since it becomes skewed for low transmission rates because of the Poisson-like statistics of the small number of electrons passing through the gating foil. For high transmission rates the distribution is Gaussian and the fitting gives the mean almost identical to the simple average.

For better illustration, though mathematically equivalent, of the reliability of the measurement even for very low transmission rates, the distribution of the average pulse height over every 300 laser shots is shown in Fig. 11(c) for a dedicated 50-minute run with $\Delta V=-15.5 \mathrm{~V}$. Also shown is the corresponding pedestal distribution obtained with the optical shutter of the laser closed: the average over every 60 shots 

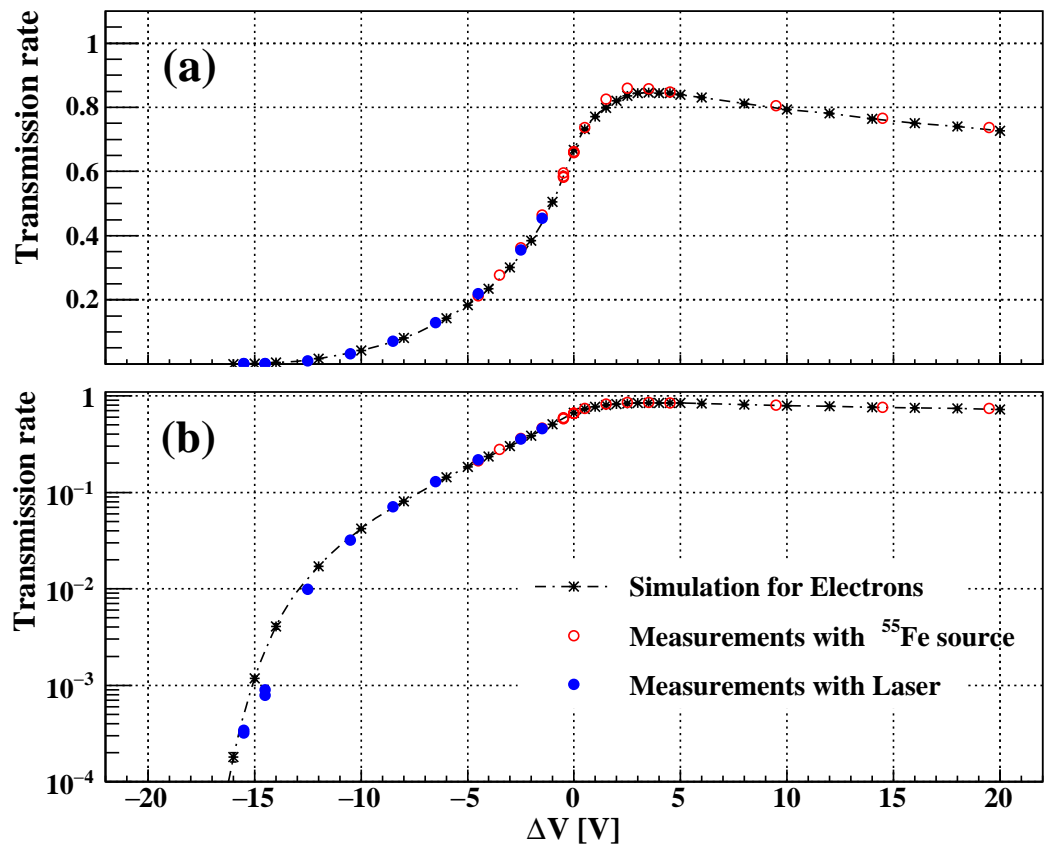

Figure 9: Measured electron transmission rate as a function of the bias voltage applied to the gating foil: (a) in linear scale, and (b) in log scale. Also shown in the figures are the measurements with the laser and the results of simulation (see Section $4.2)$.

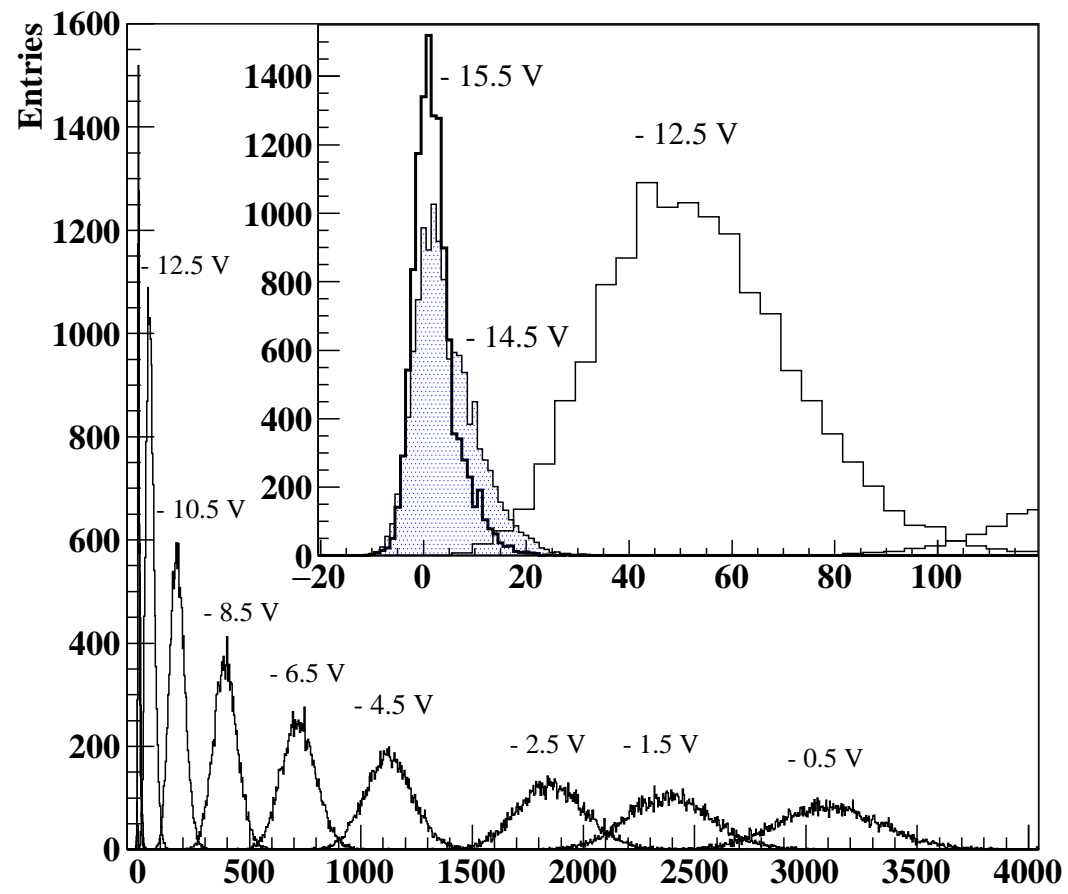

Figure 10: Examples of the pulse height distributions afterbedestats subtraction obtained with the laser for different bias voltages $(\Delta V)$ on the gating foil. A magnified view in the inset shows some of those obtained with large reverse biases. 
for a 10-minute run. The mean of a Gaussian fitted to the signal distribution is identical to the simple average of the signals ${ }^{12}$ (Fig. 11(b)). This technique allows, in principle, the measurements at higher reverse biases $(-\Delta V \mathrm{~s})$ with a larger number of laser shots, to be divided into a sufficient number of subset containing a required number $(N)$ of laser shots, as far as the laser ionization density and the gas gain of the GEM stack are stable. The mean of the average pulse height for each subset is the same as the average for single laser shots, whereas its fluctuation (the width of distribution) is reduced by a factor of $1 / \sqrt{N}$ by the central limit theorem 13 .
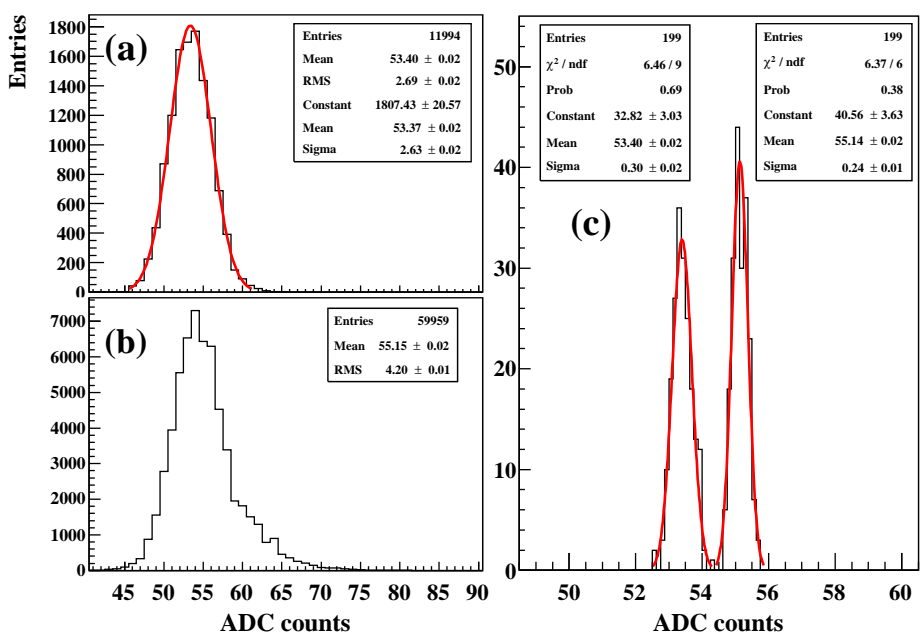

Figure 11: Pulse height distributions obtained with a set of laser runs at $\Delta V=-15.5 \mathrm{~V}$ : (a) for the pedestals obtained with the optical shutter of the laser closed in a 10-minute run, (b) for the signals in a 50-minute run, and (c) for the distribution of the pedestals (signals), averaged over every 60 (300) laser shots.

The measurements were carried out from $-0.5 \mathrm{~V}$ (for the normalization) down to $-15.5 \mathrm{~V}$. The results are shown in Fig. 9 along with those obtained by the ${ }^{55} \mathrm{Fe}$ runs. Also shown in the figure are the simulated transmission rates for electrons. The simulation was carried out using Garfield ++ (release v1r0) 332, 33. interlaced with Gmsh [34] and Elmer [35]. The microscopic tracking (AvalancheMicroscopic) was used for electrons while the Monte Carlo integration tracking (AvalancheMC) was employed for ions,

For the sake of clarity, only the simulated transmission rates are shown in Fig. 12 15 , Also shown in the figure are the transmission rates in the absence of diffusion. These are the fractions of the (inverted) electric field lines in the region upstream of the gating foil (drift region) passing through to the region downstream (transfer region). Fig. 13 shows an example of the electric field configuration in the proximity of the gating foil.

\footnotetext{
${ }^{12}$ A significant fraction ( $\gtrsim 60 \%$ ) of the signals are actually samples for which no drift electrons are detected since a single drift electron corresponds to $\sim 4$ ADC counts.

${ }^{13}$ Equivalently, the mean of the sum of pulse heights in each subset is $N$ times larger than the average pulse height for single laser shots. On the other hand, the corresponding standard deviation of the pulse height distribution increases only by a factor of $\sqrt{N}$. This is the case also for the mean and width of the corresponding pedestal distribution. As a consequence, the larger the size of subset $(N)$, the better is the apparent separation between the signal and the noise distributions.

${ }^{14}$ Results of the Monte Carlo integration tracking are identical for any singly charged positive ions, as far as they are thermal, since the diffusion constant $(D)$, both transverse and longitudinal, under an electric field $(E)$ is given by $D^{2}=$ $2 k \cdot T /(e \cdot E)$ (see Eq. (4) in Section 5.2).

${ }^{15}$ In the simulation the ions were assumed to approach the gating foil from the drift region (in the inverted electric field), whereas they cross the foil from the opposite side (transfer region) in reality. Simulations show, however, that the transmission rate of the ions (or electrons) for the flipped gating foil is almost identical to that for the present setup since the forward-backward asymmetry of its rim structure is small as compared to the distance across the hole (see Figs. 2 and 3).
} 

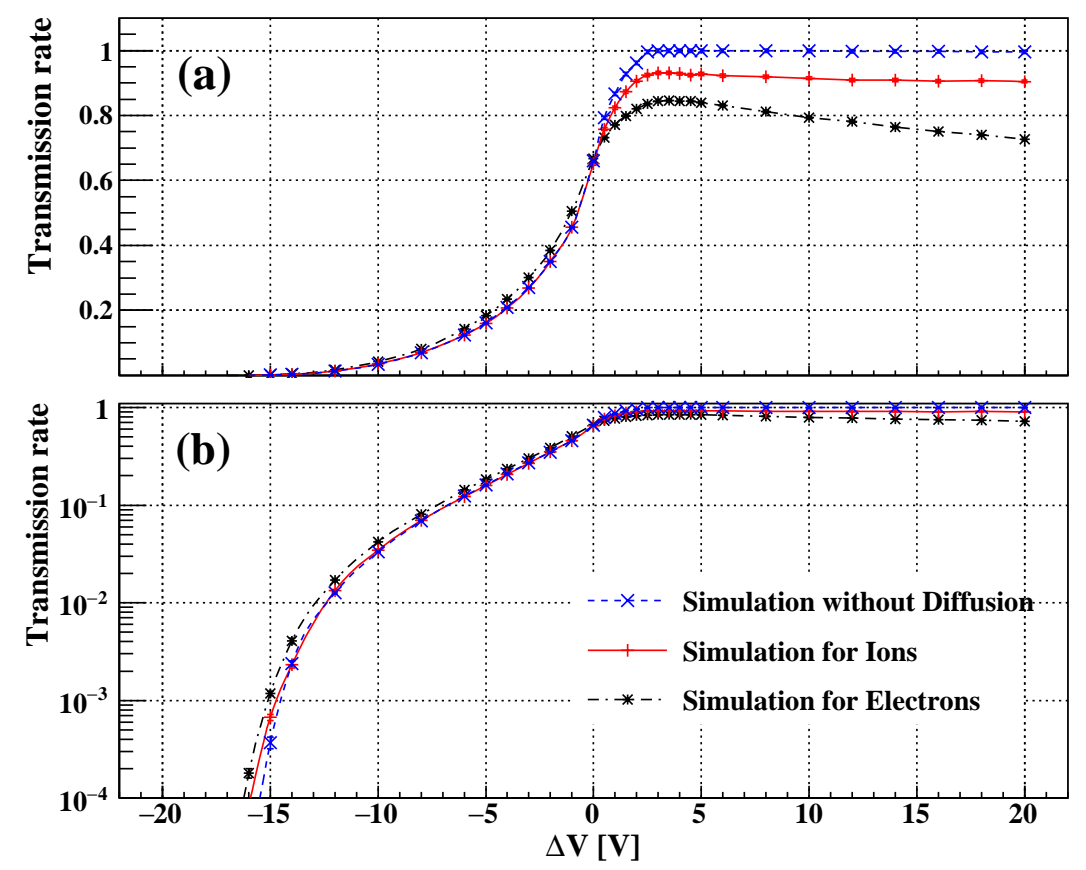

Figure 12: Simulated transmission rates for electrons and ions as function of the bias voltage applied to the gating foil: (a) in linear scale, and (b) in log scale. Also shown in the figures are the results of the simulation with diffusion switched off.

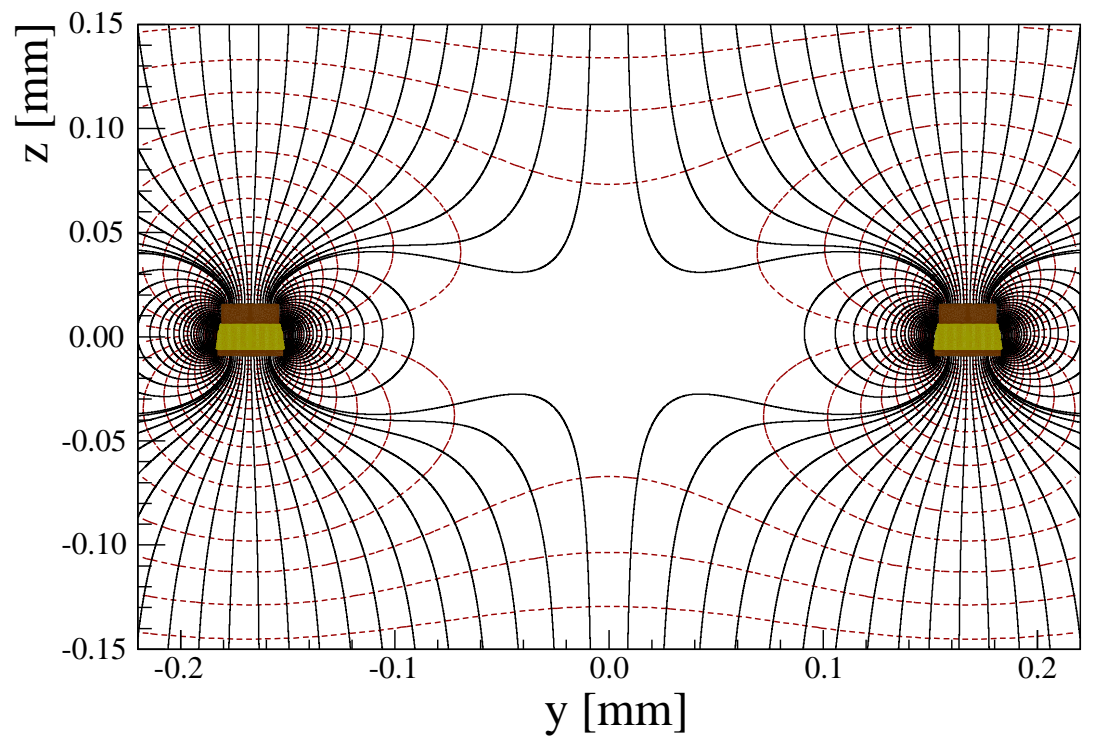

Figure 13: Electric field lines and equipotential lines around a hole in the gating foil placed in an otherwise uniform electric field of $230 \mathrm{~V} / \mathrm{cm}$, in the $y-z$ plane at $x=0$. See the left panel of Fig. 2 for the definition of coordinate system. The bias applied to the gating foil $(\Delta V)$ is $-16.0 \mathrm{~V}$. No field line penetrates the foil from the transfer region on the bottom of the figure, indicating that the foil is almost opaque in the absence of diffusion. Actually, a small part of electric field lines in the $x-z$ plane at $y=0$, for example, may penetrate the foil even at $\Delta V=-16.0 \mathrm{~V}$ because of the larger interval of the rims. The opaqueness of the foil was confirmed by tracing the drift paths of 3000000 electrons randomly placed on a virtual cathode plane in the drift region by the simulation with diffusion switched off. Not a single of them reached the transfer region. 
It is difficult to quote a reliable systematic error for each measurement of the transmission rate. The reproducibility of the measurements at several values of $\Delta V$ and the consistency between the measurements with the ${ }^{55} \mathrm{Fe}$ source and those with the laser at the same $\Delta V$ s may give estimates of the errors to be added to the relatively small statistical errors (see Table 1).

Table 1: Transmission rates measured with the ${ }^{55} \mathrm{Fe}$ source and the laser, along with statistical errors. The values obtained with the laser are normalized to the weighted average of those obtained with the ${ }^{55} \mathrm{Fe}$ source at $\Delta V=-0.5 \mathrm{~V}(0.5874 \pm$ 0.0011 , see Sections 3.2 and 4.2), and their errors include the normalization error.

\begin{tabular}{|r||l|l|l|l|}
\hline \multicolumn{1}{|c||}{$\Delta V(\mathrm{~V})$} & \multicolumn{2}{c|}{${ }^{55}$ Fe source } & \multicolumn{2}{c|}{ Laser } \\
\cline { 2 - 5 } & Transmission & Error & Transmission & Error \\
\hline 19.5 & 0.7367 & 0.0023 & - & \\
14.5 & 0.7656 & 0.0023 & - & \\
9.5 & 0.8049 & 0.0025 & - & \\
4.5 & 0.8477 & 0.0025 & - & \\
3.5 & 0.8577 & 0.0026 & - & \\
2.5 & 0.8597 & 0.0025 & - & \\
1.5 & 0.8251 & 0.0026 & - & \\
0.5 & 0.7371 & 0.0024 & - & \\
\hline 0.0 & 0.6625 & 0.0020 & & \\
0.0 & 0.6587 & 0.0020 & - & \\
0.0 & 0.6611 & 0.0019 & & \\
\hline-0.5 & 0.5953 & 0.0020 & Weighted mean & \\
-0.5 & 0.5819 & 0.0018 & 0.5874 & 0.0011 \\
-0.5 & 0.5862 & 0.0018 & Normalization & \\
\hline-1.5 & 0.4654 & 0.0015 & 0.45508 & 0.00092 \\
-2.5 & 0.3618 & 0.0012 & 0.35503 & 0.00073 \\
-3.5 & 0.2779 & 0.0011 & - & \\
-4.5 & 0.2124 & 0.0009 & 0.21895 & 0.00046 \\
-6.5 & - & & 0.12937 & 0.00033 \\
-8.5 & - & & 0.07106 & 0.00016 \\
-10.5 & - & & 0.03214 & 0.000083 \\
-12.5 & - & & 0.009915 & 0.000036 \\
\hline-14.5 & - & & 0.000895 & 0.000011 \\
-14.5 & - & & 0.000790 & 0.000012 \\
\hline-15.5 & - & & 0.0003208 & 0.0000079 \\
-15.5 & - & & 0.0003439 & 0.0000058 \\
\hline
\end{tabular}

\section{Discussion}

\subsection{Observations}

The simulation for electrons reproduces the measured transmission rates fairly well, if not perfectly, over the range of $\Delta V$ covered in the experiment. The discrepancies could be due to the slight variation of the geometry of the gating foil, the inaccuracy of the simulation, and/or unknown systematic errors in the measurements.

The simulated transmission rates for positive $\Delta V$ s are always the highest when diffusion is turned off and are always the lowest for electrons, with those for ions in-between. On the other hand, the simulated 
transmission rates for negative $\Delta V \mathrm{~s}$ are always the lowest when diffusion is turned off and are always the highest for electrons, whereas those for ions are almost identical to those without diffusion, though slightly higher. The transmission rate is not $100 \%$ for $\Delta V \gtrsim+3 \mathrm{~V}$ at which all the (inverted) field lines upstream of the foil make their way to the region downstream, and is not exactly $0 \%$ for highly negative $\Delta V \mathrm{~s}(\lesssim-16 \mathrm{~V})$ at which no electric field lines get through the gating foil ${ }^{16}$, because of diffusion, in particular for electrons.

The measured electron transmission rate is high at low forward bias voltages (positive $\Delta V \mathrm{~s}$ ) with the maximum of about $86 \%$ at $\Delta V=+3 \mathrm{~V}$ in the absence of a magnetic field. We will return to the expected electron transmission rate under a high axial magnetic field after discussions on the ion blocking power of the gating foil at reverse bias voltages in the next section.

\subsection{Ion blocking power}

The electron blocking power measured with negative $\Delta V \mathrm{~s}$ is expected to be lower than the blocking power against positive ions because of larger diffusion particularly in the vicinity of the gating foil.

For singly charged particles (electrons or ions) in thermal equilibrium with gas atoms and/or molecules, the diffusion coefficient $\left(\equiv D^{*}\right)$ and the mobility $(\mu)$ of the charged particles are related by the NernstTownsend relation (or better known as the Einstein formula) [36]

$$
\frac{e \cdot D^{*}}{\mu}=k \cdot T
$$

where $e$ is the elementary charge, $k$ represents the Boltzmann constant, and $T$, the absolute temperature of the gas. The diffusion is isotropic and the diffusion constant $D \equiv D_{\mathrm{T}}=D_{\mathrm{L}}$, with $D_{\mathrm{L}}$ being the longitudinal diffusion constant, is given by

$$
D^{2}=\frac{2 \cdot D^{*}}{W}=\frac{2 k \cdot T}{e \cdot E}
$$

where $W \equiv \mu \cdot E$ is the drift velocity of the charged particles, with $E$ being the electric field strength.

Positive ions remain thermal even in a rather high electric field because of their large masses. Therefore, Eq. (4) gives $D \sim 150 \mu \mathrm{m} / \sqrt{\mathrm{cm}}$ for ions under the drift electric field of $230 \mathrm{~V} / \mathrm{cm}$ at room temperature $(k \cdot T \sim 0.025 \mathrm{eV})$. This value for the diffusion constant of the ions is about a half of $D_{\mathrm{T}}$ for electrons measured under the same electric field in the absence of a magnetic field $(\sim 305 \mu \mathrm{m} / \sqrt{\mathrm{cm}})$, and is smaller than the simulated $D_{\mathrm{L}}$ under the same condition $(\sim 230 \mu \mathrm{m} / \sqrt{\mathrm{cm}})[11]$.

The diffusion constant of ions is proportional to $1 / \sqrt{E}$ and decreases with increasing field strength as far as they stay therma 17 . This is not the case for electrons. They are already hot in the drift region and further agitated under a higher electric field near the gating foil ${ }^{18}$. See Figs. 2 and 12 of Ref. [11], respectively for the simulated average energy, and the transverse and longitudinal diffusion constants of

\footnotetext{
${ }^{16}$ In other words, the sampling corridor for drift electrons is completely pinched off by a reverse dipole field around the hole rims (see Fig. 13).

${ }^{17}$ The back-drifting ions may contain species other than iso- $\mathrm{C}_{4} \mathrm{H}_{10}{ }^{+}$. For example, the laser photons with a wave length of $266 \mathrm{~nm}(4.66 \mathrm{eV})$ are not capable of ionizing the chamber gas by two-photon processes, indicating that trace amounts of impurities with ionization potentials lower than that of isobutane $(10.67 \mathrm{eV}$ [37]) are present in the gas. It should be noted that the argument above is valid whatever the species of positive ions are. The length of the transfer region is, however, dependent on the mobility of the impurity ions including possible products during the gas amplification process, and the duration of the beam crossing ( $\lesssim 1 \mathrm{msec}$ ). The tentative value of $\lesssim 10 \mathrm{~mm}$ assumes the mobility of $1.56 \mathrm{~cm}^{2} / \mathrm{V} / \mathrm{sec}$ for iso- $\mathrm{C}_{4} \mathrm{H}_{10}{ }^{+}$in argon [38, with an adequate safety margin.

${ }^{18}$ It should be noted that most of the electric field lines in the drift (transfer) region originate (terminate) at the narrow conducting surface on the rim of the foil, in particular when it is made almost opaque by a large reverse bias (see Fig 13).
} 
electrons in our gas mixture as function of the electric field. Consequently, the diffusion constant of ions is significantly smaller than that of electrons in the proximity, or in the holes of the gating foil. In addition, the diffusion of ions is hardly affected by the presence of a rather strong axial magnetic field $(B=3.5 \mathrm{~T}$, for example) because $D_{\mathrm{T}}(B)=D_{\mathrm{T}}(B=0) \cdot\left(1+\omega^{2} \cdot \tau^{2}\right)^{-1 / 2}$ and $\omega \cdot \tau \ll 1$ (see below).

The steady-state drift velocity $(\boldsymbol{W})$ of singly charged particles in a gas under a static electric field $\boldsymbol{E} \equiv E \cdot \hat{\boldsymbol{E}}$ and a static magnetic field $\boldsymbol{B} \equiv B \cdot \hat{\boldsymbol{B}}$, with $\hat{\boldsymbol{E}}(\hat{\boldsymbol{B}})$ being the unit vector pointing the electric (magnetic) field direction, may be given by

$$
\boldsymbol{W}=\mp \mu \cdot E \cdot \frac{1}{1+(\omega \cdot \tau)^{2}} \cdot\left(\hat{\boldsymbol{E}} \mp(\omega \cdot \tau) \cdot(\hat{\boldsymbol{E}} \times \hat{\boldsymbol{B}})+(\omega \cdot \tau)^{2} \cdot(\hat{\boldsymbol{E}} \cdot \hat{\boldsymbol{B}}) \hat{\boldsymbol{B}}\right)
$$

where $\mu \equiv e \cdot \tau / m$ is the (magnitude of) mobility and $\omega=e \cdot B / m$ is the cyclotron frequency, with $m$ being the mass and $\tau$, the mean-free-time of the charged particles 19 . This formula was derived by the approach originally adopted to Brownian motion [39]. The collective and stochastic interaction between the drifting charged particles and neutral gas atoms and/or molecules is assumed to be a viscous resistance, which is always against (anti-parallel with) the average drift velocity of charged particles $(\boldsymbol{W})$. Although this over-simplified model is not justified in general, especially for electrons in a magnetic field ${ }^{20}$, we use the equation for the qualitative argument below.

The influence of a magnetic field is negligibly small for positive ions since the value of $\omega \cdot \tau(=\mu \cdot B)$ is much smaller than unity. For example, $\omega \cdot \tau \sim 5.5 \times 10^{-4}$ for isobutane ions in our gas mixture $(\mu$ $\sim 1.56 \mathrm{~cm}^{2} / \mathrm{V} / \mathrm{sec}[38$ ]) in a magnetic field of $3.5 \mathrm{~T}$. Therefore, the positive ions move closely along the electric field lines even under a high magnetic field. As a consequence, positive ions drift like electrons in the reversed electric field and in the absence of a magnetic field, with (much) smaller diffusion. The transmission rate measured with negative $\Delta V \mathrm{~s}$ for electrons without magnetic field is hence expected to be larger than that for positive ions under an axial magnetic field of $3.5 \mathrm{~T}$.

The upper limit of the transmission rate of the gating foil for positive ions is $(3.36 \pm 0.05) \times 10^{-4}$ at $\Delta V=-15.5 \mathrm{~V}$. The blocking power for positive ions is already sufficiently high. The GEM stack (or Micromegas) is expected to be operated with an effective gas gain of $\sim 2000$ in the real experiment. The number of outgoing positive ions per incoming drift electron is about 100, assuming a value of $5 \%$ for the value of IBF of the double GEM stack. Consequently, the number of positive ions drifting back to the drift volume is expected to be at most $100 \times 3.4 \cdot 10^{-4}=0.034$ per incoming drift electron. In addition, the gating foil withstands larger negative $\Delta V \mathrm{~s}$, down at least to $-20 \mathrm{~V}$. Therefore, the average number of back-drifting positive ions in the drift region is much smaller than that of primary ions, and so would be their influence on the drift field.

\subsection{Electron transmission rate under a magnetic field}

Under a high magnetic field, the motion of drift electrons is strongly restricted to the direction of the axial magnetic field. In the drift region $(E=230 \mathrm{~V} / \mathrm{cm})$ the value of $\omega \cdot \tau=\mu \cdot B=W \cdot B / E$ in Eq. (5) is $\sim 11.6$ for $B=3.5 \mathrm{~T}$, assuming $W \equiv|\boldsymbol{W}|=7.6 \mathrm{~cm} / \mu \mathrm{sec}$ [11]. Therefore, the electron transmission rate under an axial magnetic field of $3.5 \mathrm{~T}$ is expected to be close to the optical aperture ratio of the gating foil $(\sim 82 \%)$ with $\Delta V$ around $0 \mathrm{~V}$. According to simulations, a positive value of $\Delta V$ does not improve the electron transmission rate because the slow increase of the collection efficiency is offset by the decrease in the extraction efficiency [21, 22].

\footnotetext{
${ }^{19} \mathrm{In}$ Eq. (5) the negative (positive) sign in the symbol $\mp$ is for electrons (positive ions).

${ }^{20}$ For example, Eq. (5) gives unity for the magnetic deflection coefficient $(\psi)$ [40, whereas the values of $\psi$ have been measured to be greater than unity in many gases including argon-based mixtures. See, for example, Ref. [41] for a mixture of $\operatorname{Ar}(90 \%)-\mathrm{CH}_{4}(10 \%)$.
} 
The electric field between the gating foil and the GEM stack (transfer region) was kept the same as the drift field $(230 \mathrm{~V} / \mathrm{cm})$ throughout the present experiment. A higher transmission rate of the gating foil is expected to be obtained with a higher transfer to drift field ratio under a low magnetic field because of a higher extraction efficiency of the gating foil. However, the extraction efficiency is already close to $100 \%$ under a magnetic field of $3.5 \mathrm{~T}$ with the identical electric fields for small $\Delta V \mathrm{~s}$ [22]. In addition, a higher transfer field could cause the reduction of the collection efficiency of the first stage amplification GEM.

\subsection{Electron displacement under a magnetic field}

Another issue to be addressed is the displacement (deflection) of drift electrons in the vicinity of the gating foil due to the $E \times B$ effect, which could deteriorate the azimuthal spatial resolution. A simulation shows that the displacement is small enough even at $B=3.5 \mathrm{~T}$ for small $\Delta V$ [21]. We need demonstration, however, in a strong axial magnetic field of 3-4 T. As a first step, this is to be confirmed in a beam test at $\mathrm{B}=1 \mathrm{~T}$ by measuring the spatial resolution of a large prototype TPC [16], with and without the gating foil. Deflected electrons cause the degradation of the azimuthal spatial resolution, in addition to that due to the loss of primary electrons at the gating foil. The (relative) electron transmission rate of the gating foil under a magnetic field as a function of $\Delta V$ will also be measured in the beam test by comparing the most probable pulse heights for beam particles with a well defined momentum.

\subsection{Transmission rate of unbiased gating foil}

It is interesting that the transmission rates at $\Delta V=0 \mathrm{~V}$, both measured and simulated, are close to the reduced optical transparency of the gating foil calculated assuming the rim width doubled.

An analytic calculation assuming no diffusion shows that the transmission rate of a grid consisting of equally spaced conductive wires placed in a uniform electric field is given by its optical transparency evaluated with the wire diameter doubled, in the absence of a magnetic field [42]. It is then naively expected that the transmission rate of a mesh consisting of two contiguous wire grids, which are identical and orthogonal to each other, is the square of the transmission rate of the single grid, i.e. the optical transparency of a virtual crossed mesh consisting of the wires twice as thick as the real ones. In fact, the experimental results obtained with a Frisch mesh consisting of crossed wires are consistent with the consideration above when the mesh is placed in a uniform electric field, i.e. when the field ratio $\left(E_{\mathrm{t}} / E_{\mathrm{d}}\right)$ is unity [43].

The gating foil has the rim with the aspect ratio of the cross section roughly comparable to unity and the electric field around the rim is similar to that around a conductive wire when the foil is unbiased $(\Delta V=0 \mathrm{~V})$. It is then naively expected again that the transmission rate of the gating foil is close to its reduced optical transparency evaluated with the rim width being doubled, in particular when the diffusion of drifting charged particles is small. Actually, the reduced optical transparency is well approximated by the square of the real optical aperture $\left((82 \%)^{2} \sim 67 \%\right)$ since the rim width is significantly smaller than the distance across the hole. The measured electron transmission rate at $\Delta V=0 \mathrm{~V}$ is $66.1 \pm 0.1 \%$ while the simulated values are $66.8 \pm 0.2 \%, 65.1 \pm 0.3 \%$ and $66.0 \pm 0.3 \%$, respectively for electrons, ions, and without diffusion.

\subsection{Other possible applications}

The gating foil functions also as a gate for drift electrons although it is not expected for the ILD-TPC. Therefore, it is applicable to high resolution (MPGD-based) TPCs operated in an asynchronous trigger mode of the gate [44, 45] in order to keep the ion backflow as small as possible, if the trigger rate is moderate enough to allow for the complete or sufficient collection of positive ions by the gating foil. For

this application, however, it is necessary to confirm the adequate blocking power against electrons under the axial magnetic field to be applied. 
For conventional TPCs operated in an asynchronous mode with a bipolar gating grid [46, 47], slight imbalance in the switching pulses applied on the grid wires often results in a loss of track information near the readout plane because of the settling time of the induced charge on the sense wires and the readout pads. This dead time could be comparable to the sum of the decision time for event triggers and the switching time of the gating grid itself.

The induced switching noise on the readout pads is expected to be small with the gating foil, for which a switching pulse is applied on the electrode facing the drift volume. For GEM-based TPCs the induced noise is further reduced by the GEM stack, which functions as an electrostatic shield for the pads.

The gating foil is applicable also to gaseous photo-multipliers to minimize the damage on the semitransparent photo-cathode caused by positive ion bombardment [48].

\section{Conclusion}

We have developed a real size gating foil to prevent secondary ions from entering the drift volume of the TPC for the international linear collider experiment. It is essentially a double-mesh layer with an embedded thin insulating spacer in-between and is easy to be integrated in the modularized readout unit employing a micro-pattern gas detector (MPGD) for gas amplification.

Its transparency for drift electrons in a gas mixture of $\mathrm{Ar}_{-} \mathrm{CF}_{4}(3 \%)$-isobutane $(2 \%)$ was studied using an ${ }^{55} \mathrm{Fe}$ source and a UV laser for a wide range of the bias voltages applied across the foil, under a uniform electric field of $230 \mathrm{~V} / \mathrm{cm}$ in the absence of a magnetic field.

The maximum electron transmission rate was measured to be about $86 \%$ at a forward bias voltage of $\sim+3 \mathrm{~V}$ across the foil, whereas the minimum was $(3.36 \pm 0.05$ (stat. only) $) \times 10^{-4}$ at a reverse bias voltage of $-15.5 \mathrm{~V}$. The minimum transmission rate quoted above for the electrons is the upper limit of the transmission rate for positive ions at the same reverse bias voltage, even in the presence of a magnetic field. The blocking power of the gating foil against positive ions at $\Delta V=-15.5 \mathrm{~V}$ is high enough to keep the drift region of the MPGD-based ILD-TPC virtually free from the back-drifting ions created in the gas amplification device. The maximum electron transmission rate under a $3.5 \mathrm{~T}$ magnetic field is expected to be close to the optical aperture ratio of the foil $(82 \%)$ with the foil unbiased.

We are now preparing a small chamber to be dedicated mainly to further studies on the performance of the gating foil under a reduced electronic noise condition. It includes tests under high magnetic fields up to $\sim 4 \mathrm{~T}$ in order to confirm the expected high electron transmission rate and negligible deflection of drift electrons due to the $E \times B$ effect during the passage through the gating foil. It is also planned to confirm the ion blocking capability by measuring directly the positive ion current at the cathode, and to identify the species of positive ions by measuring their mobilities. Possible charging-up effect of the gating foil will be investigated closely as well although its obvious sign was not observed throughout the present experiment.

The gating foil is also a good candidate for the gating device of (MPGD-based) TPCs operated in an asynchronous trigger mode. It can be applicable as well to gaseous photo-multipliers to protect the semi-transparent photo-cathode from back-drifting positive ions.

\section{Acknowledgments}

We are grateful to Mr. S. Otsuka and Mr. S. Watanuki with Fujikura Ltd. for the development and production of the gating foils. We would like to thank the people with REPIC Corporation for the preparation of the experimental apparatus including the prototype readout module of the ILD-TPC. We would like also to thank colleagues of the ILD-TPC collaboration for their continuous support and encouragement. This work was partly supported by the Grant-in-Aid for Specially Promoted Research Grant No. JP23000002 of Japan Society for the Promotion of Science. 


\section{Appendix A. Estimation of Fano factor and avalanche fluctuation}

In the test of the gating foil using the ${ }^{55} \mathrm{Fe}$ source, the electron transmission rate is determined from the ratio of the photo-peak positions, one for the photons converted upstream of the gating foil (gated signals) and the other for those converted downstream (ungated signals). The energy resolution for the peak corresponding to the former photons gets worse with decreasing transmission rate.

In addition to the gating or switching function, the gating foil is capable of controlling the average fraction of signal electrons to be detected after gas amplification. In this appendix the energy resolution obtained with the gating foil is analytically evaluated in terms of the Fano factor [49], the transmission rate of the gating foil, and the avalanche fluctuation in the GEM stack. The value of the Fano factor and the size of the avalanche fluctuation are then estimated from the observed resolution degradation with decreasing transmission rate 21 .

Let $n_{0}$ be the number of electrons liberated by the conversion of an X-ray photon $\left(\mathrm{Mn} \mathrm{K}_{\alpha}\right.$ or $\left.\mathrm{K}_{\beta}\right)$, and $p$ be the average transmission rate of the gating foil. In fact, $n_{0}$ fluctuates around its average $\left\langle n_{0}\right\rangle$. Then the average and the variance of the number of electrons passing through the gating foil $(n)$ are given by

$$
\begin{aligned}
\langle n\rangle & =p \cdot\left\langle n_{0}\right\rangle \\
\sigma_{n}^{2} & =\left\langle\left(n-p \cdot\left\langle n_{0}\right\rangle\right)^{2}\right\rangle \\
& =\left\langle\left(n-p \cdot n_{0}+p \cdot\left(n_{0}-\left\langle n_{0}\right\rangle\right)\right)^{2}\right\rangle \\
& =\left\langle n_{0}\right\rangle \cdot p \cdot(1-p)+p^{2} \cdot \sigma_{n_{0}}^{2} .
\end{aligned}
$$

In Eq. (A.4), a binomial distribution is assumed for the transmission through the gating foil ${ }^{22}$, and the average was taken over $n_{0}$ after averaging over $n$.

The surviving electrons are then gas-amplified by the GEM stack and detected by the readout pads. The induced charge on the pads $(Q)$ is given by

$$
Q=\sum_{i=1}^{n} q_{i}+\delta_{q}
$$

where $q_{i}$ is the gas-amplified charge of the $i$-th drift electron and $\delta_{q}$ denotes the random electronic noise charge on the pads with $\left\langle\delta_{q}\right\rangle=0$, which is uncorrelated with the signal charge. Assuming the same gas gain and its fluctuation for all the drift electrons, the average and the variance of the induced charge are given by

$$
\begin{aligned}
\langle Q\rangle & =\langle n\rangle \cdot\langle q\rangle \\
& =p \cdot\left\langle n_{0}\right\rangle \cdot\langle q\rangle \\
\sigma_{Q}^{2} & =\left\langle\left(\sum_{i=1}^{n} q_{i}+\delta_{q}-\langle n\rangle \cdot\langle q\rangle\right)^{2}\right\rangle \\
& =\left\langle\left(\sum_{i=1}^{n}\left(q_{i}-\langle q\rangle\right)+(n-\langle n\rangle) \cdot\langle q\rangle+\delta_{q}\right)^{2}\right\rangle
\end{aligned}
$$

\footnotetext{
${ }^{21}$ We found that a similar approach had already been proposed by M.A. Chefdeville for the measurement of avalanche fluctuations with Micromegas, assuming a certain value for $F$ [50].

${ }^{22}$ Actually, the transmission rate depends on the photon's conversion point relative to the hole center of the gating foil. The measured value is expected to be the average transmission since the conversion points of the uncollimated photons spread wide enough as compared to the hole pitch, and the diffusion of created electron is comparable to the size of the holes in most cases.
} 


$$
\begin{aligned}
& =\langle n\rangle \cdot \sigma_{q}^{2}+\langle q\rangle^{2} \cdot \sigma_{n}^{2}+\left\langle\delta_{q}^{2}\right\rangle \\
& =p \cdot\left\langle n_{0}\right\rangle \cdot \sigma_{q}^{2}+\langle q\rangle^{2} \cdot\left\langle n_{0}\right\rangle \cdot p \cdot(1-p)+\langle q\rangle^{2} \cdot p^{2} \cdot \sigma_{n_{0}}^{2}+\sigma_{\delta_{q}}^{2}
\end{aligned}
$$

Accordingly, the energy resolution squared $\left(R^{2}\right)$ is given by

$$
\begin{aligned}
R^{2} & \equiv \frac{\sigma_{Q}^{2}}{\langle Q\rangle^{2}} \\
& =\frac{1}{p \cdot\left\langle n_{0}\right\rangle} \cdot \frac{\sigma_{q}^{2}}{\langle q\rangle^{2}}+\frac{(1-p)}{p \cdot\left\langle n_{0}\right\rangle}+\frac{\sigma_{n_{0}}^{2}}{\left\langle n_{0}\right\rangle^{2}}+\frac{\sigma_{\delta_{q}}^{2}}{p^{2} \cdot\left\langle n_{0}\right\rangle^{2} \cdot\langle q\rangle^{2}} \\
& =\frac{1}{\left\langle n_{0}\right\rangle} \cdot\left(F-1+\frac{1+f}{p}\right)+\frac{\sigma_{\delta_{q}}^{2}}{p^{2} \cdot\left\langle n_{0}\right\rangle^{2} \cdot\langle q\rangle^{2}} \\
& =\frac{F-1}{\left\langle n_{0}\right\rangle}+\frac{1+f}{p \cdot\left\langle n_{0}\right\rangle}+\frac{\sigma_{\delta_{q}}^{2}}{\langle q\rangle^{2}} \cdot \frac{1}{p^{2} \cdot\left\langle n_{0}\right\rangle^{2}}
\end{aligned}
$$

where $F$ is the Fano factor $\left(\equiv \sigma_{n_{0}}^{2} /\left\langle n_{0}\right\rangle\right)$ and $f$ is the relative variance of gas gain for single drift electrons $\left(\equiv \sigma_{q}^{2} /\langle q\rangle^{2}\right)$.

Usually the contribution of the third term is negligibly small as far as the average transmission rate $(p)$ is not too small. In our case, the noise contribution (the width of the pedestal distribution) can be quadratically subtracted from the signal width. Therefore, the values of $F$ and $f$ are obtained respectively from the $y$-intercept and the slope of the straight line fitted through the resolution squared as a function of $1 / p$, once the value of $\left\langle n_{0}\right\rangle$ is known. An example is shown in Fig. A.1 along with a fitted straight line.

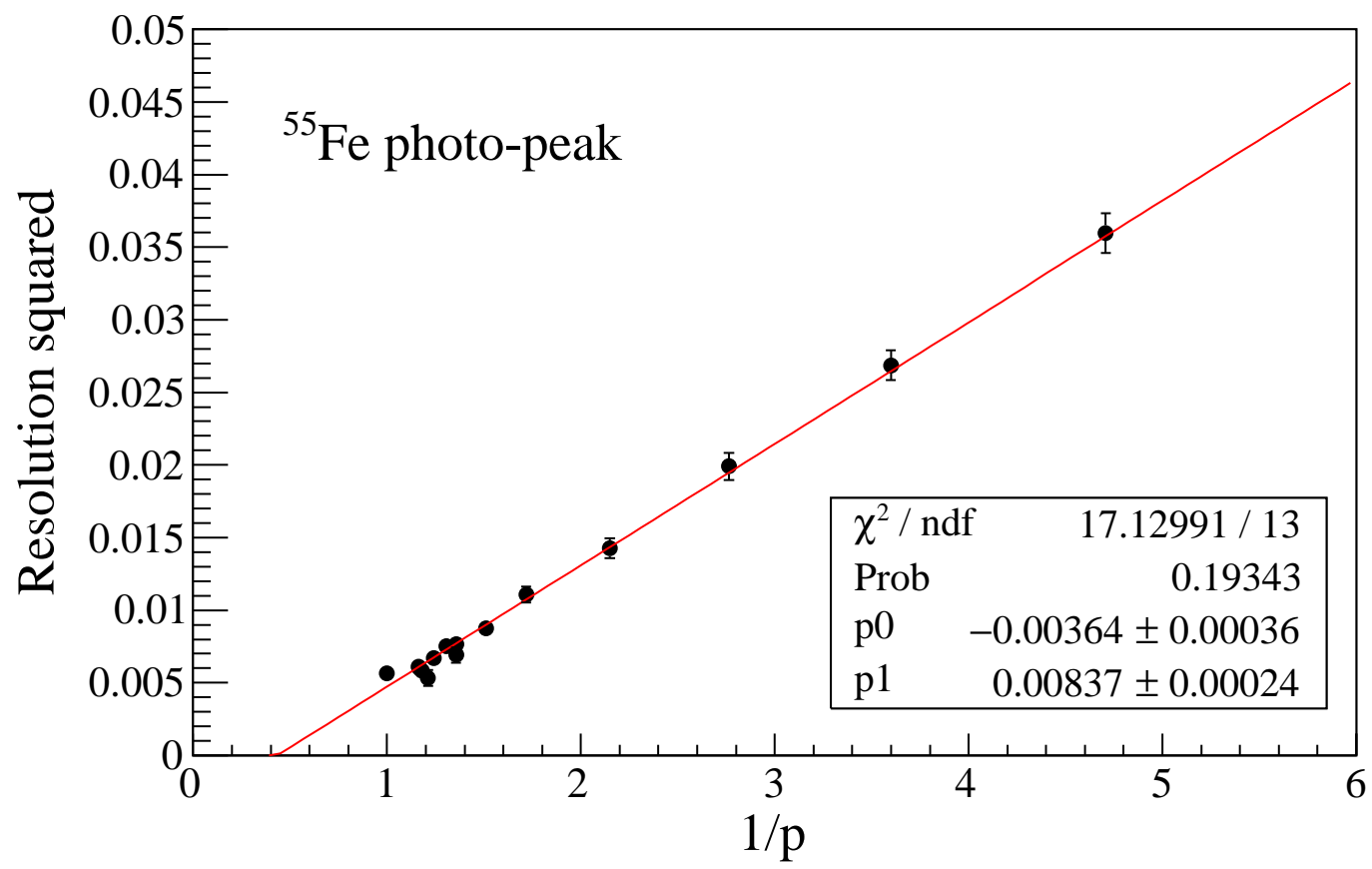

Figure A.1: Resolution squared as a function of $1 / p$ for the ${ }^{55} \mathrm{Fe}$ photo-peak for events with the signal charge deposited exclusively on the central connector. Also shown is a straight line fitted through the data points, along with the fitted parameters $\mathrm{p}_{0}$ ( $y$-intercept) and $\mathrm{p}_{1}$ (slope). The data point for $1 / p=1$ (ungated signals) was obtained with the reversed drift field. 
If we assume 220 for $\left\langle n_{0}\right\rangle{ }^{23}$ the estimated values of $F$ and $f$ are $0.20 \pm 0.08$ and $0.84 \pm 0.06$, respectively. It should be noted, however, that the values of $F$ and $f$ quoted above are preliminary since the average number of primary electrons $\left(\left\langle n_{0}\right\rangle\right)$ is not exactly known for the Penning gas mixture used ${ }^{24}$. Possible increase in the value of $\left\langle n_{0}\right\rangle$ makes $F(f)$ smaller (larger). In addition, the collection efficiency of the first GEM $\left(\equiv p^{\prime}\right)$ is not yet measured precisely. If $p^{\prime}$ is less than unity the average transmission rate $p$ in Eq. (A.15) needs to be replaced with $p \cdot p^{\prime}$ since the selections made by the gating foil and the first GEM are independent. In that case, the value of $f$ above is an overestimate, whereas that of $F$ is expected to be unaffected. It should also be noted that the local (hole-to-hole) average gain variation within a GEM foil is not taken into account in Eq. (A.15).

This technique to simultaneously measure the values of $F$ and $f$ is applicable to other gas-amplification devices such as Micromegas and/or to other gas mixtures. We plan to conduct a series of precision measurements using a dedicated small chamber, and with more statistics particularly at small transmission rates.

The energy resolution for monochromatic photons gets worse significantly with decreasing transmission rate $(p)$. From Eq. (A.15) the resolution is expressed as

$$
R^{2}=\frac{F+f}{\left\langle n_{0}\right\rangle}
$$

for the full transmission $(p=1)$, whereas for $p=0.8$, for example, it is given by

$$
R^{2}=\frac{F+0.25+1.25 \cdot f}{\left\langle n_{0}\right\rangle}
$$

when the noise contribution is negligible. If we assume the values of 0.20 and 0.84 , respectively for $F$ and $f$ the resolution degrades by $\sim 20 \%$ with the average transmission rate of $80 \%$.

In the case of specific energy loss $(\mathrm{d} E / \mathrm{dx})$ resolution for charged particles, $n_{0}$ represents the number of drift electrons detected by a pad row. Neglecting the last term, and for $p \lesssim 1$, Eq. (A.13) gives

$$
R^{2}=\frac{1+f-p+p \cdot \sigma_{n_{0}}^{2} /\left\langle n_{0}\right\rangle}{p \cdot\left\langle n_{0}\right\rangle} \sim \frac{\sigma_{n_{0}}^{2}}{\left\langle n_{0}\right\rangle^{2}}
$$

since $\sigma_{n_{0}}^{2} /\left\langle n_{0}\right\rangle \gg 1$ (see, for example, Fig. 3 in Ref. [12]). Consequently, the energy loss resolution of a single pad row is almost insensitive to the transmission rate.

In $\mathrm{d} E / \mathrm{dx}$ measurements with a TPC one usually uses the truncated (or trimmed) mean of the charges deposited on many pad rows ( $\sim 220$ in the ILD-TPC) or wires, in order to reduce unfavorable contribution from the Landau tail [55]. A simple Monte Carlo simulation shows that the energy loss resolution obtained with a truncated mean method does depend on the transmission rate, but to a significantly lesser extent as compared to that for monochromatic photons, and that the gas gain fluctuation $(f)$ has a small but detectable contribution to degrade the resolution.

The $\mathrm{d} E / \mathrm{dx}$ resolutions in the absence and presence of the gating foil will be studied in a beam test using the large prototype [16] in order to confirm the expectation above.

\footnotetext{
${ }^{23}$ In the rough estimation of $\left\langle n_{0}\right\rangle$ the weighted average energy transferred to electrons from the Mn $\mathrm{K}_{\alpha}$ and $\mathrm{K}_{\beta}$ photons was assumed to be $5.83 \mathrm{keV}$, and the $W$-values of $26.4 \mathrm{eV}, 34.3 \mathrm{eV}$ and $23.4 \mathrm{eV}$ were used respectively for Ar, $\mathrm{CF}_{4}$, and isobutane [51, 52], without consideration for their stopping powers.

${ }^{24}$ In fact, larger values of $\left\langle n_{0}\right\rangle$ have been observed by measurements with pixel readout for argon-isobutane mixtures (see, for example, Refs. [53, 54]).
} 


\section{References}

[1] David R. Nygren, Proposal to investigate the feasibility of a novel concept in particle detection, LBL internal note, February 1974, https://inspirehep.net/record/1365360.

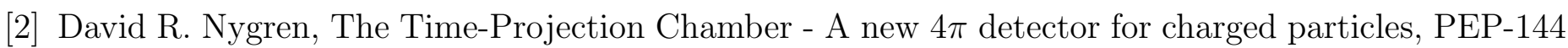
in the proceedings of the 1974 PEP summer study, http://lss.fnal.gov/conf/C740805/p58.pdf.

[3] Dave Nygren, The Time-Projection Chamber - 1975, PEP-198 in the proceedings of the 1975 PEP summer study, http://slac.stanford.edu/pubs/slacreports/reports04/slac-r-190.pdf.

[4] F. Sauli, Nuclear Instruments and Methods in Physics Research A 386 (1997) 531, https://doi.org/10.1016/S0168-9002(96)01172-2.

[5] Y. Giomataris, et al., Nuclear Instruments and Methods in Physics Research A 376 (1996) 29, https://doi.org/10.1016/0168-9002(96)00175-1.

[6] T. Behnke, J.E. Brau, P.N. Burrows, J. Fuster, M. Peskin, M. Stanitzki, Y. Sugimoto, S. Yamada, H. Yamamoto (eds.), The International Linear Collider Technical Design Report - Volume 4: Detectors (2013), arXiv:1306.6329, https://arxiv.org/ftp/arxiv/papers/1306/1306.6329.pdf.

[7] The International Linear Collider Technical Design Report (2013), http://www. linearcollider.org/ILC/Publications/Technical-Design-Report.

[8] K. Ackermann, et al., Nuclear Instruments and Methods in Physics Research A 623 (2010) 141, https://doi.org/10.1016/j.nima.2010.02.175.

[9] Makoto Kobayashi, Nuclear Instruments and Methods in Physics Research A 562 (2006) 136, https://doi.org/10.1016/j.nima.2006.03.001.

[10] D.C. Arogancia, et al., Nuclear Instruments and Methods in Physics Research A 602 (2009) 403, https://doi.org/10.1016/j.nima.2009.01.014.

[11] M. Kobayashi, et al., Nuclear Instruments and Methods in Physics Research A 641 (2011) 37, https://doi.org/10.1016/j.nima.2011.02.042.

[12] Makoto Kobayashi, Nuclear Instruments and Methods in Physics Research A 729 (2013) 273, https://dx.doi.org/10.1016/j.nima.2013.07.028.

[13] M. Kobayashi, et al., Nuclear Instruments and Methods in Physics Research A 767 (2014) 439, https://doi.org/10.1016/j.nima.2014.08.027.

[14] R. Yonamine, et al., Journal of Instrumentation 9 (2014) C03002, https://doi.org/10.1088/1748-0221/9/03/C03002.

[15] M. Dixit, et al., Nuclear Instruments and Methods in Physics Research A 581 (2007) 254, https://doi.org/10.1016/j.nima.2007.07.099. 
[16] D. Attié, et al., Nuclear Instruments and Methods in Physics Research A 856 (2017) 109, https://doi.org/10.1016/j.nima.2016.11.002.

[17] Chris Adolphsen, et al. (eds.), The International Linear Collider Technical Design Report - Volume 3: Accelerator Part II: Baseline Design (2013), arXiv:1306.6328, https://arxiv.org/ftp/arxiv/papers/1306/1306.6328.pdf.

[18] A. Vogel, Beam-induced backgrounds in detectors at the ILC, PhD Thesis, Universität Hamburg, 2008, http://www-library.desy.de/preparch/desy/thesis/desy-thesis-08-036.pdf.

[19] T. Yamashita, et al., Nuclear Instruments and Methods in Physics Research A 283 (1989) 709, https://doi.org/10.1016/0168-9002(89)91445-9.

[20] F. Sauli, L. Ropelewski, P. Everaerts, Nuclear Instruments and Methods in Physics Research A 560 (2006) 269, http://doi.org/10.1016/j.nima.2005.12.239.

[21] P. Gros, et al., Journal of Instrumentation 8 (2013) C11023, https://doi.org/10.1088/1748-0221/8/11/C11023.

[22] Katsumasa Ikematsu (on behalf of the LCTPC-Japan Collaboration), Development of Large-Aperture GEMs as a Gating Device of the ILC-TPC for Blocking Positive Ion Feedback, IEEE Nuclear Science Symposium Conference Record (2014) N44-7, https://doi.org/10.1109/NSSMIC.2014.7431237.

[23] Peter Schade, Jochen Kaminski, for the LCTPC Collaboration, Nuclear Instruments and Methods A 628 (2011) 128, and references cited therein, https://doi.org/10.1016/j.nima.2010.06.300.

[24] C. Ligtenberg, et al., Nuclear Instruments and Methods A 908 (2018) 18, and references cited therein, https://doi.org/10.1016/j.nima.2018.08.012.

[25] H.J. Hilke, Nuclear Instruments and Methods 217 (1983) 189, https://doi.org/10.1016/0167-5087(83)90130-8.

[26] Anna Peisert, Nuclear Instruments and Methods 217 (1983) 229, https://doi.org/10.1016/0167-5087(83)90139-4.

[27] D. Arai, et al., Development of Gating Foils To Inhibit Ion Feedback Using FPC Production Techniques, Proceedings of the 4th Conference on Micro-Pattern Gaseous Detectors, https://agenda.infn.it/getFile.py/access? contribId=60\&sessionId=2\&resId=1\& materialId=paper\&conf $I d=8839$.

Also https://agenda.infn.it/getFile.py/access? contribId=60\&sessionId=2\&resId=0\& material Id=slides\&conf $I d=8839$ for the presentation slides.

[28] M. Kobayashi, et al., Nuclear Instruments and Methods in Physics Research A 845 (2017) 236, https://doi.org/10.1016/j.nima.2016.06.073. 
[29] A. Oskarsson, K. Dehmelt, J-P. Dewulf, X. Janssen, A. Junique, L. Jonsson, G. De Lentdecker, B. Lundberg, U. Mjornmark, L. Musa, E. Stenlund, G. Trampitsch, R. Volkenborn, Y. Yang, L. Osterman, A General Purpose Electronic readout system for tests of Time Projection Chambers, equipped with different avalanche multiplication systems, Eudet-Memo 2008-49,

http://www . eudet.org/e26/e28/e615/e830/eudet-memo-2008-49.pdf.

[30] V. Hedberg, et al., Journal of Instrumentation 10 (2015) C01035, https://doi.org/10.1088/1748-0221/10/01/C01035.

[31] T. Tamagawa, et al., Nuclear Instruments and Methods in Physics Research A 608 (2009) 390, https://doi.org/10.1016/j.nima.2009.07.014.

[32] S.F. Biagi, Nuclear Instruments and Methods in Physics Research A 421 (1999) 234, https://doi.org/10.1016/S0168-9002(98)01233-9.

[33] https://garfieldpp.web.cern.ch/garfieldpp/.

[34] Christophe Geuzaine and Jean-Françoi Remacle, International Journal for Numerical Methods in Engineering 79 (11) (2009) 1309, https://onlinelibrary.wiley.com/doi/epdf/10.1002/nme.2579.

[35] https://www.csc.fi/web/elmer/.

[36] L.G.H. Huxley, R.W. Crompton, The Diffusion and Drift of Electrons in Gases, Wiley, New York (1974) (Chapter 1 and Chapter 3), and references cited therein.

[37] L.G. Christophorou, Atomic and Molecular Radiation Physics, Wiley-Interscience (1971).

[38] G. Schultz, G. Charpak, F. Sauli, Mobilities of positive ions in some gas mixtures used in proportional and drift chambers,

Revue de Physique Appliquée (Paris), 1977, 12 (1), pp.67-70, https://doi.org/10.1051/rphysap:0197700120106700, also https://hal.archives-ouvertes.fr/jpa-00244121/document.

[39] P. Langevin, Sur la théorie du mouvement brownien, Les Comptes Rendus de l'Académie des sciences 146 (1908) 530.

Also, D.S. Lemons and A. Gythiel, On the Theory of Brownian Motion,

American Journal of Physics 65 (1997) 1079 for the translated version with an introduction, https://doi.org/10.1119/1.18725.

[40] K. Hoshina, et al., Nuclear Instruments and Methods in Physics Research A 479 (2002) 278, Section 5.1 , and references cited therein, https://doi.org/10.1016/S0168-9002(01)00908-1.

[41] Thomas Kunst, Bernhardt Gotz, Bernhard Schmidt, Nuclear Instruments and Methods in Physics Research A 324 (1993) 127, https://doi.org/10.1016/0168-9002(93)90971-J.

[42] O. Bunemann, T.E. Cranshaw, J.A. Harvey, Design of Grid Ionization Chambers, Canadian Journal of Research Vol.27, Sec. A (1949) 191, http://www.nrcresearchpress.com/doi/pdf/10.1139/cjr49a-019. 
[43] R. Bevilacqua, et al., Nuclear Instruments and Methods in Physics Research A 770 (2015) 64, https://doi.org/10.1016/j.nima.2014.10.003.

[44] W. Blum, W. Riegler, L. Rolandi, Particle Detection with Drift Chambers, Springer-Verlag (2008), Chapter 9.

[45] H.J. Hilke, Time projection chambers, Reports on Progress in Physics, 73 (2010) 116201, Section 3.2.3,

https://doi.org/10.1088/0034-4885/73/11/116201.

[46] A. Breskin, et al., Nuclear Instruments and Methods 178 (1980) 11, https://doi.org/10.1016/0029-554X(80)90853-8.

[47] Peter Némethy, Piermaria J. Oddone, Nobukazu Toge, Akira Ishibashi, Nuclear Instruments and Methods 212 (1983) 273, https://doi.org/10.1016/0167-5087(83)90702-0.

[48] D. Mörmann, A. Breskin, R. Chechik, D. Bloch, Nuclear Instruments and Methods in Physics Research A $516(2004) 315$ https://doi.org/10.1016/j.nima.2003.08.156.

[49] U. Fano, Physical Review 72 (1947) 26, https://journals.aps.org/pr/pdf/10.1103/PhysRev.72.26.

[50] M. Chefdeville, A proposal to study gas gain fluctuations in Micromegas detectors, 2009, http://lappweb.in2p3.fr/ chefdevi/Work_LAPP/Gain_fluctuations/proposal_gain_ fluctuations.pdf.

[51] International Commission on Radiation Units and Measurements, Average Energy Required to Produce an Ion Pair, ICRU Report No. 31, Washington DC (1979).

[52] G.F. Reinking, L.G. Christophorou, S.R. Hunter, Journal of Applied Physics 60 (1986) 499, https://doi.org/10.1063/1.337792.

[53] D. Attié, et al., Study of avalanche fluctuations and energy resolution with an InGrid-TimePix detector, talk presented at 12th Vienna Conference on Instrumentation (VCI 2010), https://indico.cern.ch/event/51276/contributions/2034170/attachments/967018/ 1373280/2_Colas.pdf.

[54] Jochen Kaminski, et al., GridPix detector with Timepix3 ASIC, talk presented at the 5th International Conference on Micro-Pattern Gas Detectors (MPGD 2017), https://indico.cern.ch/event/581417/contributions/2522462/attachments/1465797/ 2265982/GridPix_TP3.pdf.

[55] W.W.M. Allison and J.H. Cobb, Relativistic Charged Particle Identification by Energy Loss, Annual Review of Nuclear and Particle Science 30 (1980) 253, and references cited therein, https://doi.org/10.1146/annurev.ns.30.120180.001345. 